\title{
Pareto Optimal Security Resource Allocation for Internet of Things
}

\author{
ANTONINO RULLO and DANIELE MIDI, Purdue University \\ EDOARDO SERRA, Boise State University \\ ELISA BERTINO, Purdue University
}

\begin{abstract}
In many Internet of Thing (IoT) application domains security is a critical requirement, because malicious parties can undermine the effectiveness of IoT-based systems by compromising single components and/or communication channels. Thus, a security infrastructure is needed to ensure the proper functioning of such systems even under attack. However, it is also critical that security be at a reasonable resource and energy cost. In this article, we focus on the problem of efficiently and effectively securing IoT networks by carefully allocating security resources in the network area. In particular, given a set of security resources $\mathcal{R}$ and a set of attacks to be faced $\mathcal{A}$, our method chooses the subset of $\mathcal{R}$ that best addresses the attacks in $\mathcal{A}$, and the set of locations where to place them, that ensure the security coverage of all IoT devices at minimum cost and energy consumption. We model our problem according to game theory and provide a Pareto-optimal solution in which the cost of the security infrastructure, its energy consumption, and the probability of a successful attack are minimized. Our experimental evaluation shows that our technique improves the system robustness in terms of packet delivery rate for different network topologies. Furthermore, we also provide a method for handling the computation of the resource allocation plan for large-scale networks scenarios, where the optimization problem may require an unreasonable amount of time to be solved. We show how our proposed method drastically reduces the computing time, while providing a reasonable approximation of the optimal solution.
\end{abstract}

CCS Concepts: • Security and privacy $\rightarrow$ Formal security models; Distributed systems security; Mobile and wireless security;

Additional Key Words and Phrases: Internet of things, stochastic allocation, pareto analysis, clustering

\section{ACM Reference format:}

Antonino Rullo, Daniele Midi, Edoardo Serra, and Elisa Bertino. 2017. Pareto Optimal Security Resource Allocation for Internet of Things. ACM Trans. Priv. Secur. 20, 4, Article 15 (October 2017), 30 pages.

https://doi.org/10.1145/3139293

The work reported in this article has been partially funded by NSF under grant no. ACI-547358 and by the Technological District on Cyber Security PON Project (grant no. PON03PE_00032_2), funded by the Italian Ministry of University and Research.

Authors' addresses: A. Rullo, (current address) DIMES Department, Universita della Calabria, 87036 Rende, Italy; email: n.rullo@dimes.unical.it; D. Midi and E. Bertino, Lawson Computer Science Department, Purdue University, West Lafayette, IN 47906, USA; email: \{dmidi, betrtino\}@purdue.edu; E. Serra, Department of Computer Science, Boise State University, Boise, ID 83725, USA; email: edoardoserra@boisestate.edu.

Permission to make digital or hard copies of all or part of this work for personal or classroom use is granted without fee provided that copies are not made or distributed for profit or commercial advantage and that copies bear this notice and the full citation on the first page. Copyrights for components of this work owned by others than ACM must be honored. Abstracting with credit is permitted. To copy otherwise, or republish, to post on servers or to redistribute to lists, requires prior specific permission and/or a fee. Request permissions from permissions@acm.org.

(C) 2017 ACM 2471-2566/2017/10-ART15 \$15.00

https://doi.org/10.1145/3139293

ACM Transactions on Privacy and Security, Vol. 20, No. 4, Article 15. Publication date: October 2017. 


\section{INTRODUCTION}

The term Internet of Things (IoT) refers to the interconnection of small devices able to interact with each other and cooperate to accomplish common tasks. Many kinds of systems and technologies fall under the definition of IoT: identification and tracking technologies, wired and wireless sensor and actuator networks, enhanced communication protocols and distributed intelligence for smart objects, home automation, and smart city applications (Atzori et al. 2010). It is critical that an effective security infrastructure be adopted to ensure the integrity and reliability of exchanged data even under attack. Besides effectiveness, another important requirement for such an infrastructure is to carefully address security resource efficiency, as an infrastructure with very high demands in terms of additional resources for security would not be viable. Its cost would in fact outweigh the low cost of IoT technologies. Despite the fact that IoT networks can differ from each other in several aspects, such as topology, mobility, size, degree of heterogeneity, and location, they also have some common characteristics that can be leveraged in the design process of a security infrastructure. They are prone to physical attacks and eavesdropping, since they are often unattended and typically communicate via wireless channels. They make use of (battery powered) devices with low computational power. They share known software and hardware vulnerabilities, and, as a consequence, also many kinds of security systems, such as intrusion detection and prevention systems. Based on such common features, it is possible to design a general method that works for different IoT scenarios for designing an effective and efficient ad hoc security infrastructure. Both efficiency and effectiveness depend on the choice of the security tools (IDS, special hardware, additional devices, etc.), and how they are allocated in the system of interest. Among all possible security resource allocation plans, some are more efficient-as they require a lower amount of energy consumption and/or entail a cheaper cost in terms of additional equipment, while other plans are more effective-because harder to be evaded.

In this article, we focus on the problem of effectively and efficiently securing IoT networks and propose a method to compute a resource allocation plan as a Pareto-optimal solution. In particular, given a set of security resources $\mathcal{R}$ and a set of attacks to be faced $\mathcal{A}$, our method chooses the subset of $\mathcal{R}$ that best addresses the attacks in $\mathcal{A}$, and the set of locations where to place them, that ensure a certain security level at minimum cost and energy consumption. We start from the assumption that, given a set of available security tools, we can build a security resource as a combination of one or more tools, and we can estimate its installation cost and its average energy consumption at operating speed. We can thus estimate the efficiency of an allocation plan, based on the total energy consumption of the security infrastructure and the costs of its components. To measure the effectiveness of an allocation plan, we use two metrics, namely risk and criticality. The former is defined as the maximum number of network nodes that are no longer protected when an attacker succeeds in taking down at least one security resource. The latter is a measure of how critical certain nodes are for the correct operation of the network. We identify a set of heuristics for computing the criticality value of each node of the IoT network of interest. We model the interaction between attacker and defender as a Stackelberg leadership model in which the leader (the defender) moves before the follower (the attacker) (von Stackelberg et al. 2010).

We show how to compute a Pareto-optimal defender strategy (resource allocation plan) in two steps. First, among all possible allocation plans, we compute the subset of the Pareto-optimal plans, as the solutions of a three-objective optimization problem, which minimize (i) the total energy consumption, (ii) the installation cost, and (iii) the maximum criticality. In the second step, we select the plan, among those identified in the previous step, which minimizes the risk-as the solution of a single-objective optimization problem. The resulting defender strategy is efficiency optimal, because it entails the lowest energy consumption and the cheapest installation cost, while 
its effectiveness lies in the fact that the number of security resources an attacker needs to take down is maximized-and, consequently, the probability of a successful attack is reduced.

For large instances, the optimization problem might require an unreasonable amount of time to be solved. To overcome this limitation, we propose a divide-et-impera approach that consists of decomposing the original problem in smaller sub-problems that are easier and faster to solve. To this end, we first divide the network area in smaller sub-areas with the help of a $k$-medoids clustering algorithm. Then we solve in parallel the related sub-problems, and, finally, we merge the partial solutions.

We have implemented and tested our algorithms with different network topologies. Our results show that the proposed method provides security infrastructure options for IoT networks that represent different combinations of energy consumption, cost, and probability of successful attacks. Further, we show how our proposed divide-et-impera approach drastically reduces the computing time providing a reasonable approximation of the optimal solution.

\section{THREAT MODEL}

In the threat model assumed in our work, the attacker may perform attacks at different layers (Sharma and Ghose 2010). While our approach is independent from the actual attack, we assume that the attacker can take any of the steps commonly used to carry out attacks, such as physically tampering with the network nodes; capturing and reprogramming legitimate nodes and security resources; adding malicious entities to the network to overhear data communications; injecting false data and control traffic; intercepting and drop data packets; introducing interference; claiming multiple identities; exfiltrating data, security credentials, or encryption keys; compromising IoT devices with the help of a malicious one, and redirecting data flows. All these malicious actions are at the basis of known attacks that undermine different security aspects of an IoT application. Attacks are classified according to the confidentiality, integrity, authentication, and availability (CIAA) model: Confidentiality means the protection of data information and exchanges, integrity involves the protection of data from unauthorized modification, authentication ensures that the network components are who they claim to be prior to exchanging information, and availability requires that forwarding services and information exchanges are accessible for the network components or external actors. The confidentiality aspect concerns eavesdropping attacks, where the goal of the attacker is to obtain information about the exchanged data. Integrity is impacted when a legitimate node or legitimate traffic is corrupted, for example, routing tables, data packets, and so on. Authentication attacks occur when an attacker masquerades as a legitimate user identity. Availability attacks (DoS) attempt to make services unavailable for a period of time by attacking the power supply, storage media, or network, and availability can be denied. Furthermore, we make the following two assumptions about the attacker: (s)he needs to compromise at least one security resource to carry out an attack, and (s)he is aware of the security resources employed by the defender and plays her/his best strategy, that is, chooses to compromise the security resource that maximizes his/her chances of performing a successful attack. In case of an attacker behaving differently than the worst-case scenario (therefore, in some sense, less smartly), the allocation provided by our approach will clearly be able to provide an even higher security than that estimated at the time of the plan computation. For the attackers able to compromise all security resources, no defender strategy is able to protect the network against. The strategy that best addresses such a situation is the one that delays the attack as much as possible by maximizing the number of security resources the attacker needs to compromise for launching the attack. This way, the defender can hope that the attacker will bail out, because the "pre-attack" process is much too long or complicated or that (s)he will be caught while trying to compromise the security resources. The most effective way to implement such a strategy is to deploy a highly redundant security infrastructure 
to make any attack very hard to be carried out. However, this solution is not efficient in terms of energy consumption and can also be quite expensive for large-scale networks. Our approach, instead, provides the most effective solution among the most efficient ones. While this scenario is out of scope for our work, it would be nevertheless easy to add additional redundant resources on top of the computed optimal plan, in particular around the areas of the network deemed more important by the administrator.

\section{SECURITY MODEL AND DEFINITIONS}

The goal of the defender is to secure the network. To do so, the defender needs to choose a set of security resources among the available ones and decide where to deploy them in the network area for securing all the network nodes. Due to the heterogeneity of IoT systems, there are different kinds of security tools the defender may have to use. Intrusion detection systems (IDSs) (Raza et al. 2013) as well as intrusion prevention systems (IPSs) (Roman et al. 2011) can be installed on network nodes to, respectively, detect ongoing and prevent future attacks; physical tools, like directional antennas and highly sensitive transceivers (Godara 1997; Nasipuri and Li 2002), can be installed on watchdogs nodes, thus enabling these nodes to better control other nodes (Tumrongwittayapak and Varakulsiripunth 2009); nodes with tamper-resistant hardware can replace normal network nodes to strengthen the network (Brasser et al. 2015); nodes with specialized hardware can be used to implement specific security techniques (Čapkun et al. 2003; Hu et al. 2003); and additional nodes can be deployed at specific locations in the network with the sole purpose of executing security tasks (Tumrongwittayapak and Varakulsiripunth 2009). Furthermore, there are several characteristics of IoT that can be leveraged to design a security resource well fit for IoT. While heterogeneous, most IoT devices communicate and operate on standard mediums and protocols, such as IEEE 802.15.4 (IEEEE 2007), WiFi or Bluetooth for mediums, and Alliance Zigbee (2006) or 6LoWPAN (Hui et al. 2009) for protocols. Therefore, as long as a device communicates by using any of these mediums and protocols, effective techniques such as promiscuous overhearing and watchdog-based mechanisms (Huang and Lee 2003; Mishra et al. 2004) can be deployed (see e.g. Midi et al. (2017)). Notice that a security resource may embed one or more of these security tools or may just collect data to be sent to security services located in remote locations.

As an example, let consider a smart street lighting system with the aim of optimizing energy and maintenance costs, covering an area of thousands of square meters and consisting of hundreds of lamps. In this scenario, a security manager needs to compute a security resource allocation plan by which (i) the resources are placed on locations from where they are able to monitor/defend all the network devices (lamps) from possible attackers, and (ii) the minimum number of resources are allocated to minimize the cost and/or the total consumption of energy (when the security resources are power constrained devices, as it may be the case of many IoT scenarios). Furthermore, either the type of security resources to be employed and their allocation in the network area depend on the type of attacks that have to be faced. For instance, a security resource consisting of a tamperresistant hardware would address attackers with the intent of tampering with the lamps, while a security resource consisting of an IDS would be more appropriate for addressing attackers with the aim of injecting false data to perform a DoS. In those cases, security resources would be placed differently according to their functionality (given by the embedded tools), and a security manager would choose one of them (or both), depending on the attack(s) he/she want to address.

\subsection{Basic Concepts and Notation}

Here we introduce the basic concepts and notations that help us to formalize the IoT environment and the security requisites for the defender. 
Definition 3.1 (Security Resource). A security resource is a tuple $s r=\langle c, e, t, l o c\rangle$, where:

$-c$ is the cost;

$-e$ is the energy consumption;

$-t$ is the resource's type;

$-l o c$ is the location. ${ }^{1}$

The value of the attribute $t$ (type) depends on the set of embedded security tools, so security resources with different functions will be of different type.

Definition 3.2 (Network). A network is an undirected graph $N=\langle V, E\rangle$, where

$-V=\{(e, l o c, c r): \mathrm{e}$ is the node energy consumption, loc is the node location, and $\mathrm{cr}$ is the criticality value $\}$ is the set of network nodes;

$-E=\left\{\left\{v_{1}, v_{2}\right\}: v_{1}\right.$ is in the communication range of $v_{2}$, and vice versa, and $\left.v_{1}, v_{2} \in V\right\}$ is the set of links.

We define a network as an undirected graph, because we consider the physical-based topology, that is, the one inferred by the communication range of network nodes, different from the routingbased topology, which is always a sub-graph of the physical one, whatever routing protocol is adopted, and whatever is the direction of the links. Thus, also devices directly connected to the Internet are considered part of a network if they fall in the communication range of other devices.

We use the notation $v \cdot x$ and sr.y to denote attributes $x$ and $y$, of node $v$ and and resource $s r$, respectively. Given a network $N$ and a set of security resources $\mathcal{R}$, we define:

$-A P \subseteq \mathcal{R}$ as a resource allocation plan for $N$;

$-\mathcal{A P}$ as the set of all possible resource allocation plans for $N$;

- the criticality of a network node $n \in N$ as the measure of its relevance for the correct function of the network (examples can be found in the rest of the article);

- function crit : $\mathcal{R} \times \mathcal{A P} \times N \rightarrow \mathbb{N}$ as the criticality associated with a security resource $s r$; given an allocation plan $A P \in \mathcal{A P}$ and a network $N$, crit $(s r, A P, N)$ returns the maximum criticality value over the set of nodes of $N$ that are not any longer protected if $s r$ stops working and no other security resource $s r^{\prime} \in A P$ covers them;

- function risk : $\mathcal{R} \times \mathcal{A P} \times N \rightarrow \mathbb{N}$ as the risk associated with a resource sr; given an allocation plan $A P \in \mathcal{A P}$ and a network $N$, risk $(s r, A P, N)$ returns the number of nodes of $N$ that are not any longer protected if $s r$ stops working and no other security resource $s r^{\prime} \in A P$ covers them.

- the function Neighbors $: V \rightarrow 2^{V}$, such that Neighbors $(v)=\left\{v^{\prime}:\left\{v, v^{\prime}\right\} \in E\right\}$;

-the function Edges $: V \rightarrow 2^{E}$, such that $\operatorname{Edges}(v)=\left\{\left\{v, v^{\prime}\right\}: v^{\prime} \in \operatorname{Neighbors}(v)\right\}$;

$-\mathcal{L}=L_{V} \cup L_{A}$ as the set of locations, where $L_{V}$ is the set of locations taken by each $v \in V$, and $L_{A}$ is the set of all other available locations in the network area;

- the function Res : $2^{\mathcal{L}} \rightarrow 2^{\mathcal{R}}$, such that, given a set of locations $X, \operatorname{Res}(X)=\{s r: s r$.loc $\in X\}$, that is, the resources that can be placed on locations in $X$;

- the domain of a security resource $s r$ as the set of locations that are in the action range of $s r$, that is, the portion of network area covered by $s r$;

-the function Dom $: 2^{\mathcal{R}} \rightarrow 2^{\mathcal{L}}$, such that given a set of resources $X, \operatorname{Dom}(X)$ returns the set of locations that belong to the domain of all $s r \in X$;

$-T=\{s r . t: s r \in \mathcal{R}\}$ as the set of security resources types.

\footnotetext{
${ }^{1}$ The attribute $l o c$ in Definition 3.1 helps us to simplify the formalization of the linear programs we show hereafter. The basic idea is the following: given that in the network area there are many locations where a security resource can be placed, for each resource $s r^{*}$ we assume to have $s r_{1}, \cdots s r_{n}$ resources, one for each location where $s r^{*}$ can be located.
} 


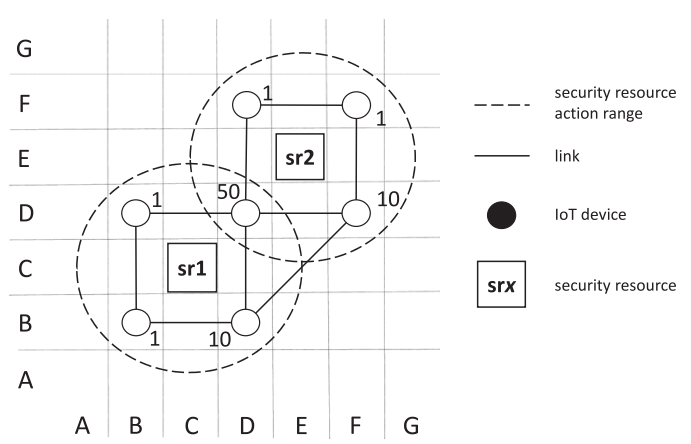

Fig. 1. An example network: the main square represents the network area; locations of the network area are represented by internals dotted squares.

Example 3.3. Figure 1 shows a network with seven nodes protected by two security resources $s r_{1}$ and $s r_{2}$. The number associated to each node denotes the node's criticality value (here the criticality is proportional to the number of links that involve a node).

If one of the two security resources stops working, then the set of nodes left without protection is $S_{1}=\{a, c, d\}$ for $s r_{1}$ and $S_{2}=\{e, f, g\}$ for $s r_{2}$. The node with maximum criticality in $S_{1}$ is node $d$ (criticality=10), while in $S_{2}$ is node $g$ (criticality=10). The criticality and risk values of the two security resources, thus, are $\operatorname{crit}\left(s r_{1}, A P, N\right)=\operatorname{crit}\left(s r_{2}, A P, N\right)=10$ and $\operatorname{risk}\left(s r_{1}, A P, N\right)=$ $\operatorname{risk}\left(s r_{2}, A P, N\right)=\left|S_{1}\right|=\left|S_{2}\right|=3$, with $A P=\left\{s r_{1}, s r_{2}\right\}$. Furthermore, we have that

$$
\begin{aligned}
& - \text { Neighbors }(a)=\{c, b\}, \text { Neighbors }(e)=\{f, b\}, \ldots ; \\
& - \text { Edges }(a)=\{\{a, c\},\{a, b\}\}, \text { Edges }(e)=\{\{e, b\},\{e, f\}\}, \ldots ; \\
& -\mathcal{L}=\left\{\left\langle x_{1}, x_{2}\right\rangle: x_{1}, x_{2} \in\{A, B, C, D, E, F, G\}\right\} ; \\
& -L_{V}=\{\langle B, B\rangle,\langle B, D\rangle,\langle D, B\rangle,\langle D, D\rangle,\langle D, F\rangle,\langle F, F\rangle\langle F, D\rangle\} ; \\
& -L_{A}=\mathcal{L} \backslash L_{V} ; \\
& -\operatorname{Res}(\langle C, C\rangle)=\left\{s_{1}\right\}, \operatorname{Res}(\langle E, E\rangle)=\left\{s r_{2}\right\}, \operatorname{Res}(\langle C, C\rangle,\langle E, E\rangle)=\left\{s r_{1}, s r_{2}\right\} ; \\
& -\operatorname{Dom}\left(s r_{1}\right)=\left\{\left\langle x_{1}, x_{2}\right\rangle: x_{1}, x_{2} \in\{B, C, D\}\right\}, \operatorname{Dom}\left(s r_{2}\right)=\left\{\left\langle x_{1}, x_{2}\right\rangle: x_{1}, x_{2} \in\{D, E, F\}\right\} .
\end{aligned}
$$

The idea behind the notion of risk lies in the fact that the minimization of the maximum risk restricts the operating range of the attacker, in case (s)he manages to compromise a security resource. Thus, (s)he probably will need to compromise more than one resource to carry out the attack. For instance, during a sybil attack (Sharma and Ghose 2010) a malicious node presents multiple identities to other nodes. The attack is much more effective when those identities belong to real nodes of the network. Thus, the attacker needs to compromise a certain number of nodes, from which to steal the identity, before the one from which to start the attack. However, if the defender strategy is well designed, the attacker will probably have to damage more than one security resource to steal a sufficient number of identities, therefore delaying the attack and increasing the risk of being detected. Other types of attacks, instead, need just to compromise one node. This is the case of the black hole attack (Sharma and Ghose 2010), in which the compromised node drops all the incoming packets. A black hole is much more effective when the attacker chooses to compromise a critical node, such as one with a high incoming traffic rate. In this case, a defender strategy must provide stronger coverage for the most critical nodes, that is, those that are more relevant for the correct function of the network. An attacker will thus be forced to compromise more than one resource to attack the most critical nodes. 


\subsection{Definition of Secure Network}

A formal definition of secure network that matches the techniques adopted by the security systems is crucial, since it gives the guidelines for the formalization of the linear constraints we will use in the Pareto analysis. We can classify security systems into two main categories: detection and prevention systems. Those categories correspond to two different security policies: (i) node/link monitoring and (ii) node/link hardening. As an example of link monitoring we mention the IoT IDS by Raza et al. (2013), which checks the link quality for the detection of network layer and routing attacks, and the Liteworp system (Khalil et al. 2005) that uses guard nodes as a countermeasure for wormhole attacks in WSNs. We would like also to mention Dataguard (Zhang and Liu 2010) as an example of node monitoring based on a code attestation technique to check the presence of malicious code in the memory of network nodes. Titan (Brasser et al. 2015) is an example of a security architecture for hardening tiny devices with a hardware-assisted dynamic root of trust. The encryption key management scheme by Eschenauer and Gligor (2002), to harden links among nodes neighbors, is an example of link hardening. According to those two security policies, we derive the definition of secure network.

Definition 3.4 (Secure Link). A link $\left\{v, v^{\prime}\right\}$ is secure if at least one of the following conditions holds:

$(\sigma)$ both nodes $v$ and $v^{\prime}$ are in the communication range of the same watchdog;

$(\gamma)$ both nodes $v$ and $v^{\prime}$ can establish a secure communication channel.

Definition 3.5 (Secure Node). A node $v$ is secure if at least one of the following conditions holds:

$(\alpha) \quad v$ is tamper resistant;

$(\beta)$ every link that involves $v$ is secure.

In condition $(\alpha)$ the term tamper resistant means that the node is equipped with a security tool that makes it inaccessible to attackers wishing to compromise it. In other words, Definition 3.5 states that the correct functioning of a node can be guaranteed by avoiding malicious code injection, or by monitoring/strengthening the links connecting to the other nodes. In Definition 3.4, a watchdog (Marti et al. 2000) is a security resource that monitors network nodes behavior. Past approaches to intrusion detection use watchdogs for overhearing in/out-coming traffic of neighbors nodes, performing code attestation, checking the signal strength, and so on. Conditions $(\alpha)$ and $(\gamma)$ capture the policy adopted by prevention systems (node/link hardening), while conditions $(\beta)$ and $(\sigma)$ the policy adopted by detection systems (node/link monitoring).

We can now define a network $N=\langle V, E\rangle$ as secure if each node $v \in V$ is secure, according to Definition 3.5.

\section{PLAYERS' STRATEGY}

In this section, we define the concept of "strategy" for the defender and the attacker. Their interaction is modeled as a Stackelberg game (von Stackelberg et al. 2010). In such a game the defender plays the leader and makes the first move by installing a security infrastructure $A P$. The attacker plays the follower by trying to compromise one or more security resources $s r \in A P$, so the attack can be carried out on the nodes that are no longer protected by the damaged security resources.

\subsection{Defender's Strategy}

The defender strategy consists of a security resource allocation plan. Given that each security resource entails an installation cost and some energy consumption, the best resource allocation plan for the defender is the one that provides a reasonable balance between efficiency, in terms of 
energy consumption and cost, and effectiveness, in terms of maximum risk and maximum criticality. The best plan is computed in two steps. In the first step we perform a Pareto analysis that solves the optimization problem defined by the following equation:

$$
\min _{A P \in \mathcal{A P} P}\left\{e c(A P), t c(A P), \max _{s r \in A P} \operatorname{crit}(s r, A P, N)\right\},
$$

where $e c(A P)$ and $t c(A P)$ denote the total energy consumption and the total cost of the allocation plan $A P$, respectively, and $\max _{s r \in A P} \operatorname{crit}(s r, A P, N)$ is the maximum criticality value over the set of nodes of $N$ that are no longer protected when one resource in $A P$ stops working. The Pareto analysis consists in computing a set of Pareto points $p=(e c, t c, c r)$, which we refer to as Pareto curve. Each point corresponds to a set of allocation plans, that is, all the plans (strategies) that have an energy consumption, a total cost, and a maximum criticality equal to the values of $e c$, $t c$ and $c r$, respectively. Then, we choose the point $p^{+}$whose values best fit our efficiency and criticality requirements. In the second step, we compute the best allocation plan (best defender strategy) by solving the optimization problem defined by the following equation:

$$
\min _{A P \in A P^{+}}\left\{\max _{s r \in A P} \operatorname{risk}(s r, A P, N)\right\},
$$

where $A P^{+} \subseteq \mathcal{A P}$ is the set of allocation plans that entail an energy consumption, a cost, and a maximum criticality as the values of the Pareto point $p^{+}$chosen in the previous step; and $\max _{s r \in A P} \operatorname{risk}(s r, A P, N)$ is the maximum number of nodes of $N$ that remain unprotected when a security resource in $A P$ stops working.

\subsection{Attacker's Strategy}

We assume that an attacker needs to compromise at least one security resource to carry out an attack. In fact, whatever is the attack, an attacker always needs to first open a breach in the security, before attacking the system. We address the worst case attacker, that is the one who knows the defenders strategy $A P$, and plays her/his best strategy, that is, chooses to compromise the security resource that maximizes the risk (i.e., leaves unprotected the maximum number of nodes), the criticality (i.e., leaves unprotected the most critical nodes), or a combination of both. An attacker strategy is represented by a security resource $s r \in A P$. The best attacker strategy is defined as follows:

where $\alpha+\beta=1$.

$$
s r^{*}=\max _{s r \in A P} \alpha \cdot \operatorname{risk}(s r, A P, N)+\beta \cdot \operatorname{crit}(s r, A P, N)
$$

\section{COMPUTING THE DEFENDER'S STRATEGY}

In this section, we first introduce a heuristic for computing node's criticality, and then we briefly survey the concept of Pareto analysis. Finally, we describe the two steps for computing the optimal defender strategy.

\subsection{Computing Node's Criticality}

Computing the criticality of each node is a key aspect of our approach. The more accurately we identify the "relevance" of each node for the correct function of the network, the better the defender strategy works. Due to the heterogeneous nature of IoT devices and IoT networks, it is not possible to design a general method for computing nodes criticality that works well for all IoT scenarios. Rather, we emphasize that one has to follow an ad hoc approach for each specific case. However, we propose a two-steps approach to determine criticality that we believe can be adopted for the majority of the existent IoT scenarios. The first step assigns a criticality value to each node based on the rate of their in/out-going traffic. Nodes that are more fundamental for the correct 
propagation of data will have higher criticality. The criticality values are then normalized across all the nodes. This information can be automatically collected by means of actual traffic analysisif the network has already been deployed-or through simulations of data communications on the topology and chosen protocol-if the network is still in its design stage. In the second step the network administrators can tune any criticality value by either (i) overriding the computed value with a new, custom one or (ii) by specifying "multipliers" for specific areas of the network, to be applied to all the nodes located in that area. The second step is not required. However, our two-step approach helps in characterizing the network in the best possible way by leveraging the administrator's knowledge of the intrinsics of the network and its functionality, while still automating most of the activities for characterizing the node criticality. Our two-step approach is well suited to many IoT scenarios. First, the correct transmission of data is often at the core of the security goal. In these cases, the first step of our approach properly addresses the needs to distinguish nodes basing on their in/out-going traffic rate. Second, many IoT networks are designed with a hierarchical structure, and thus some nodes need to be considered at a different level of criticality with respect to the other ones. For instance, cluster-head nodes in cluster-based network topologies, that are responsible of the correct functioning of nodes belonging to their cluster. Or, in home IoT environments as in many WSN applications, it is often the case that external attackers launch attacks trough the router linking the IoT network with the Internet. In such cases, it is mandatory to assign the highest criticality value to the router, which represents an access point for intruders. In cases in which network components/devices are all of the same type, they communicate in a peer-to-peer fashion, and they belong to a closed network (i.e., not connected with the Internet), a security manager might want to consider them equally critical, thus making the criticality a metric that does not affect the computation of the allocation plan.

\subsection{Overview of the Pareto Analysis}

Pareto analysis (Messac et al. 2003) is a classic optimization method used in situations in which there are multiple competing objectives that must somehow be satisfied simultaneously. The basic idea behind Pareto optimization of three competing objective functions $\phi_{1}, \phi_{2}$, and $\phi_{3}$ subject to a set $C$ of constraints is as follows. Suppose that $\sigma=\left(x_{1}, x_{2}, x_{3}\right)$ and $\sigma^{\prime}=\left(x_{1}^{\prime}, x_{2}^{\prime}, x_{3}^{\prime}\right)$ are two different solutions (resource allocation plans) and suppose that $\phi_{1}, \phi_{2}$, and $\phi_{3}$ are minimization problems. We say that $\sigma$ dominates $\sigma^{\prime}$, denoted $\sigma \triangleright \sigma^{\prime}$, iff:

$$
\left(x_{1} \leq x_{1}^{\prime} \wedge x_{2} \leq x_{2}^{\prime} \wedge x_{3} \leq x_{3}^{\prime}\right) \wedge\left(x_{1}<x_{1}^{\prime} \vee x_{2}<x_{2}^{\prime} \vee x_{3}<x_{3}^{\prime}\right)
$$

A solution $\sigma$ is said to be Pareto optimal w.r.t. a set of minimization problems $\Phi$ and constraints $C$ if and only if there is no solution $\sigma^{\prime} \neq \sigma$ such that $\sigma^{\prime} \triangleright \sigma$. We use Pareto analysis to solve the optimization problem defined by Equation (1) to find a compromise strategy. The main point of this method is the computation of the Pareto curve (see Messac et al. (2003)).

\subsection{First Step: Pareto Analysis for the Defender}

To perform our Pareto analysis, let

$$
\mathcal{P}=\left\{\left(e c(A P), t c(A P), \max _{s r \in A P} \operatorname{crit}(s r, A P, N)\right) \mid A P \in \mathcal{A P}\right\}
$$

be the set of all possible values for our three objectives.

Definition 5.1 (Pareto Curve). The Pareto curve PC for the three-objective optimization problem defined by Equation (1) is the set $\left\{(a, b, c) \mid(a, b, c) \in \mathcal{P}\right.$ and $\nexists\left(a^{\prime}, b^{\prime}, c^{\prime}\right)$ such that $\left(a^{\prime}, b^{\prime}, c^{\prime}\right) \triangleright$ $(a, b, c)\}$. 


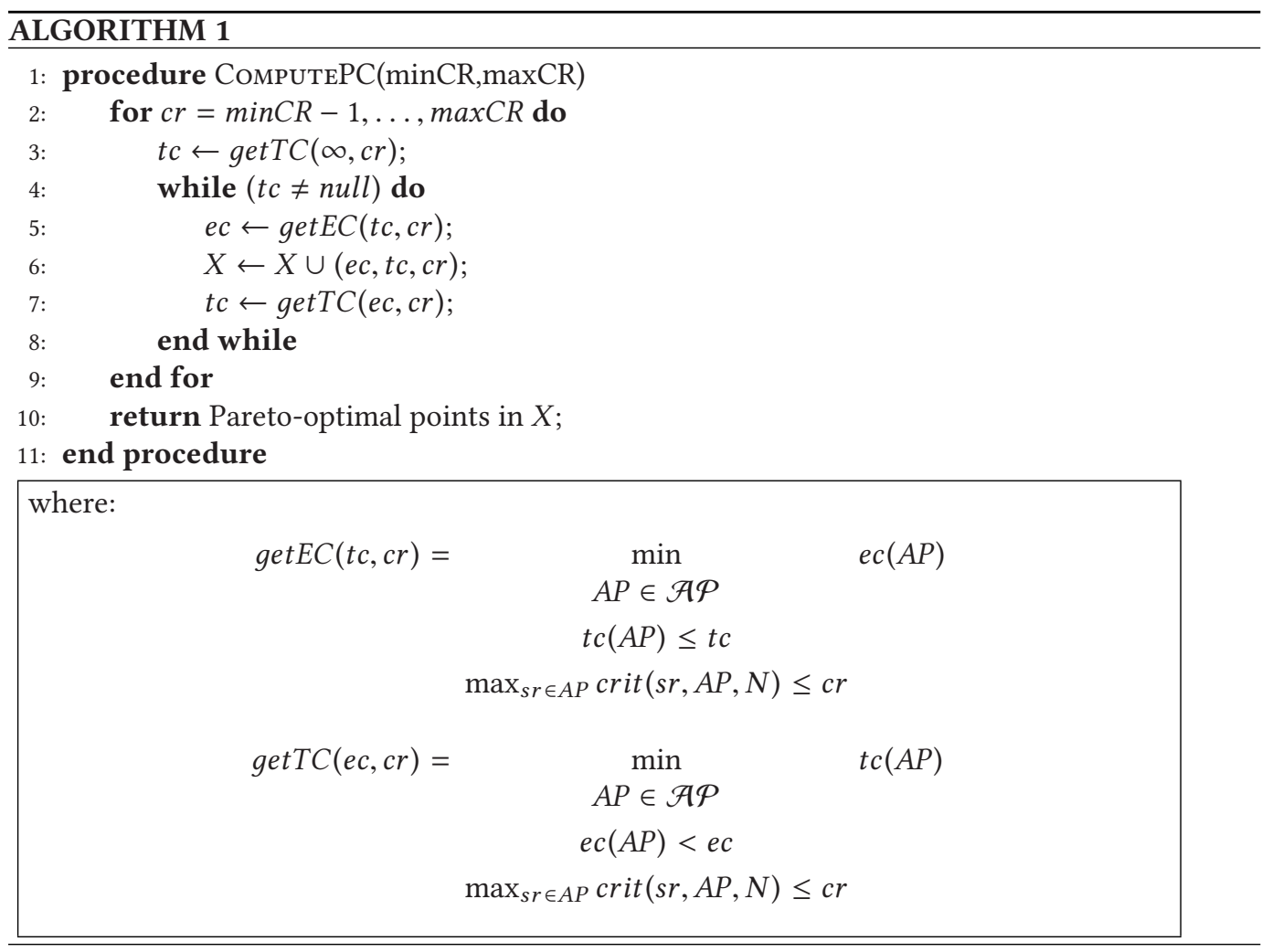

Algorithm 1 computes the Pareto curve of the three-objective optimization problem defined by Equation (1). More specifically, by fixing each possible value of criticality $\mathrm{cr}$ (Line 2), the algorithm computes the set $X$ of points $p=(e c, t c, c r)$ (Lines 3-8), where $e c$ and $t c$ are the total energy consumption and the total cost, respectively. The first step is to compute the minimum total cost without considering the energy consumption (Line 3). This optimization is performed by the function getTC, and the value is assigned to the variable $t c$. Then, given $t c$ and $c r$, function getEC computes the minimum total energy consumption ec (Line 5), and the point (ec, tc, $c r)$ is added to $X$ (Line 6). Now the algorithm computes a different value of $t c$ (Line 7). This value is obtained by the function getTC, which minimizes the total cost by imposing that the total energy consumption is strictly less than the one previously computed. If such value exists, then the algorithm continues to generate more points by iterating in the while loop; otherwise, it assigns $t c=$ null and exits the loop. Finally, in Line 10 the algorithm returns all Pareto (non-dominated) points of $X$. Notice that in Line 2, we start to enumerate the values of $c r$ from $\min C R-1$, so, as no node exists with $c r<\min C R$, the plan being calculated with $c r=\min C R-1$ provides $r i s k=0$, that is, each network node is covered by at least two security resources. For allocation plans with $c r=0$, it is not necessary to perform the second optimization step, since we already know that the risk will be also equal to 0. An example of Pareto curve can be found in Example 3 of Section 6. It can be noticed that no point $(e c, c, c r)$ of the curve is dominated, according to the definition given in Section 5.2 .

THEOREM 5.2. If there exists a polynomial output algorithm computing the Pareto curve, then $\mathbf{P}=$ NP. 
Proof. We demonstrate the NP-hardness of our problem with a reduction to the MinimumSeTCover problem, which is proved to be NP-hard. The MinimumSetCover problem is as follows: Given a set of elements $X$, a set $Y$ of subsets of $X$, and an integer $k$, determine whether there exists a subset $H \subseteq Y$ s.t. $|H| \leq k$ and $X=\cap_{S \in Y} S$. The reduction is the following:

- for each element $x$ in $X$, we have a network node $n_{x}$ and a security resource $s r_{x}$ that protects $n_{x}$ only;

- for each element $y$ in $Y$, we have a security resource $s r_{y}$ that protects the network node $n_{x}$ only, s.t. $x \in X$.

- the cost of security resources $s r_{x}$ is 0 , while that of security resources $s r_{y}$ is 1 ;

- the energy consumption for all the security resources is 0 , while the criticality for the nonprotected network nodes is 1 .

If $k^{\prime}$ is the minimum cost when the criticality is 0 , according to the reduction, then $k^{\prime}$ is the cardinality of the minimum set $H$ that is able to cover all the elements in $X$.

\subsection{Linear Constraints for the Pareto Analysis}

We formalize the set of basic constraints as follows.

\subsubsection{Variables.}

$-x_{1}, \ldots, x_{|\mathcal{R}|}$; if resource $s r_{i}$ belongs to $A P$, then $x_{i}=1$, otherwise $x_{i}=0$;

$-z_{11}, \ldots, z_{\lceil|\mathcal{L}||\mathcal{R}|\rceil}$; if location $l o c_{i} \in \operatorname{Dom}\left(s r_{j}\right)$ and $s r_{j} \in A P$, then $z_{i j}=1$, otherwise $z_{i j}=0$;

$-q_{1}, \ldots, q_{|V|}$; if $l o c_{i} \in L_{V}$ belongs to the domain of no more then one security resource, then $q_{i}=1$; otherwise, $q_{i}=0$;

$-l_{1}, \ldots, l_{|E|}$; if link $e_{i}$ is secure, then $l_{i}=1$; otherwise, $l_{i}=0$.

All variables are binary.

5.4.2 Basic Constraints. The set of basic constraints is shown in Figure 2. C.4 says that no more than one security resource can be placed on the same location. ${ }^{2}$ C. 5 says that variable $z_{i j}$ is assigned value 1 if $s r_{j} \in A P$ and $l o c_{i} \in \operatorname{Dom}\left(s r_{j}\right)$. C.6 and C.7 capture the fact that, if a location $l o c_{i} \in L_{V}$ is guarded by no more than one security resource, then $q_{i}=1$; otherwise, $q_{i}=0$. Here $M$ is a constant big enough to satisfy the constraints for all values of $q_{i}$ and the left-hand side of the equation (any integer greater than $\mathcal{R}$ ). Variables $q_{i}$ are used to compute the risk and the criticality associated with each resource $s r \in A P$. C.8 captures the definition of secure node, according to which a node is secure if conditions $(\alpha)$ or $(\beta)$ of Definition 3.5 hold, that is, expressions $\sum_{s r_{j} \in \operatorname{Res}\left(v_{i} . l o c\right): s r_{j} \in T_{1}} x_{j}$ or $c_{i}$, respectively, equal 1 . The value of variable $c_{i}$ is computed by constraints 9 and 10 , and it has value equal to 1 if all links belonging to $\operatorname{Edges}\left(v_{i}\right)$ are secure, otherwise it is equal to 0 . The definition of secure link is captured by C.11, according to which a link is secure $\left(l_{k}=1\right)$ if conditions $(\sigma)$ or $(\gamma)$ of Definition 3.4 hold, that is, variables $a_{k}$ or $b_{k}$ equal 1 . The values of these variables are computed by C.12-15 and C.16-17, respectively.

C.18 says that for tamper resistant nodes the variable $q_{i}$ is forced to 0 , that is, it does not affect the risk and criticality values of the allocation plan is being computed. Finally, C.19 says that each location has to belong to the domain of at least one security resource in $A P$. This last constraint is fundamental, because it addresses the dynamic characteristic of IoT networks. In an IoT environment, as new devices can enter and leave the network area, it is important to monitor all locations where any new device can move over time. When this happens, we are sure to have at least one security resource monitoring its location. We emphasize that the concept of dynamic

\footnotetext{
${ }^{2}$ C.4 does not imply any loss of generality, since a security resource can embed more than one security tool.
} 


$$
\begin{aligned}
& \sum_{s r_{i} \in \operatorname{Res}\left(l o c_{j}\right)} x_{i} \leq 1, \quad \forall l o c_{j} \in \mathcal{L} \\
& z_{i j}=x_{j}, \quad \forall s r_{j} \in \mathcal{R}, \forall l o c_{i} \in \operatorname{Dom}\left(s r_{j}\right) \\
& \sum_{s r_{j}: l o c_{i} \in \operatorname{Dom}\left(s r_{j}\right)} z_{i j} \leq q_{i}+M \cdot\left(1-q_{i}\right), \quad \forall l o c_{i} \in L_{V} \\
& \sum_{s r_{j}: l o c_{i} \in \operatorname{Dom}\left(s r_{j}\right)} z_{i j} \geq 2-q_{i}, \quad \forall l o c_{i} \in L_{V} \\
& \sum_{s r_{j} \in \operatorname{Res}\left(v_{i} . l o c\right): s r_{j} . t \in T_{\alpha}} x_{j}+c_{i} \geq 1, \quad \forall v_{i} \in V \\
& \sum_{e_{k} \in \operatorname{Edges}\left(v_{i}\right)} l_{k}<c_{i}+\mid \text { Neighbors }\left(v_{i}\right) \mid, \quad \forall v_{i} \in V \\
& \sum_{e_{k} \in \operatorname{Edges}\left(v_{i}\right)} l_{k} \geq c_{i} \cdot\left|\operatorname{Neighbors}\left(v_{i}\right)\right|, \quad \forall v_{i} \in V \\
& l_{k} \geq a_{k}, \quad l_{k} \geq b_{k}, \quad l_{k} \leq a_{k}+b_{k}, \quad \forall e_{k} \in E \\
& \sum_{s r_{j}:\left\{v . l o c, v^{\prime} . l o c\right\} \in \operatorname{Dom}\left(s r_{j}\right) \wedge s r_{j} . t \in T_{\sigma}} d_{k j} \geq a_{k}, \quad \forall\left\{v, v^{\prime}\right\}_{k} \in E \\
& a_{k} \geq d_{k j}, \quad \forall\left\{v, v^{\prime}\right\}_{k} \in E, \forall s r_{j}:\left\{v . l o c, v^{\prime} . l o c\right\} \in \operatorname{Dom}\left(s r_{j}\right) \wedge s r_{j} . t \in T_{\sigma} \\
& \sum_{l o c_{i} \in\left\{v . l o c, v^{\prime} . l o c\right\}} z_{i j} \geq 2 d_{k j}, \quad \forall\left\{v, v^{\prime}\right\}_{k} \in E, \forall s r_{j}:\left\{v . l o c, v^{\prime} . l o c\right\} \in D o m\left(s r_{j}\right) \wedge s r_{j} . t \in T_{\sigma} \\
& \sum_{l o c_{i} \in\left\{v . l o c, v^{\prime} . l o c\right\}} z_{i j}<d_{k j}+2, \quad \forall\left\{v, v^{\prime}\right\}_{k} \in E, \forall s r_{j}:\left\{v . l o c, v^{\prime} . l o c\right\} \in D o m\left(s r_{j}\right) \wedge s r_{j} . t \in T_{o} \\
& \sum_{l o c_{i} \in\left\{v . l o c, v^{\prime} . l o c\right\}} z_{i j} \geq 2 b_{k}, \quad \forall\left\{v, v^{\prime}\right\}_{k} \in E, \forall s r_{j}:\left\{v . l o c, v^{\prime} . l o c\right\} \in D o m\left(s r_{j}\right) \wedge s r_{j} . t \in T_{\gamma} \\
& \sum_{l o c_{i} \in\left\{v . l o c, v^{\prime} . l o c\right\}} z_{i j}<b_{k}+2, \quad \forall\left\{v, v^{\prime}\right\}_{k} \in E, \forall s r_{j}:\left\{v . l o c, v^{\prime} . l o c\right\} \in D o m\left(s r_{j}\right) \wedge s r_{j} . t \in T_{\gamma} \\
& z_{i j}+q_{i} \leq 1, \quad \forall l o c_{i} \in L_{V}, \forall s r_{j} \in \mathcal{R}: s r_{j} . t \in T_{\alpha} \wedge s r_{j} . l o c=l o c_{i} \\
& \sum_{s r_{j}: l o c_{i} \in \operatorname{Dom}\left(s r_{j}\right)} z_{i j} \geq 1, \quad \forall l o c_{i} \in \mathcal{L}
\end{aligned}
$$

Fig. 2. Basic constraints.

topology we refer to is different from the concept of mobility. In our network model, it is expected that the nodes are static and that the topology changes due to the (dis)appearance of nodes. For mobile device scenarios, we refer the reader to Rullo et al. (2017b). In C.8 and C.14-18 the symbols $T_{\alpha}, T_{\sigma}$, and $T_{\gamma}$ are subset of $T$, the set of security resource types. In particular, $T_{\alpha}$ is the set of types of security resources that make a node tamper resistant, $T_{\sigma}$ is the set of types of security resources that behave as watchdogs, and $T_{\gamma}$ is the set of types of security resources that make a node able to establish a security communication channel with its neighbors. In Section 6, we implement the proposed method for two real-case IoT scenarios where we show how to compute such sets.

5.4.3 Linear Programs. The basic constraints capture the dependencies between the variables in the integer linear programs shown in Figure 3.

The objective function of the formulation in Figure 3(top) says that we want to minimize the total energy consumption of the security infrastructure. Constraint 20 says that the total cost must be lower or equal to $t c$, and Constraint 21 says that the criticality value of the nodes protected by no more than one security resource must not exceed $\mathrm{cr}$. The objective function of the linear program in Figure 3(center) minimizes the total cost of the set of security resources. Constraint 22 imposes that the total energy consumption be strictly less than $e c$, while Constraint 23 is equal to Constraint 21 . 


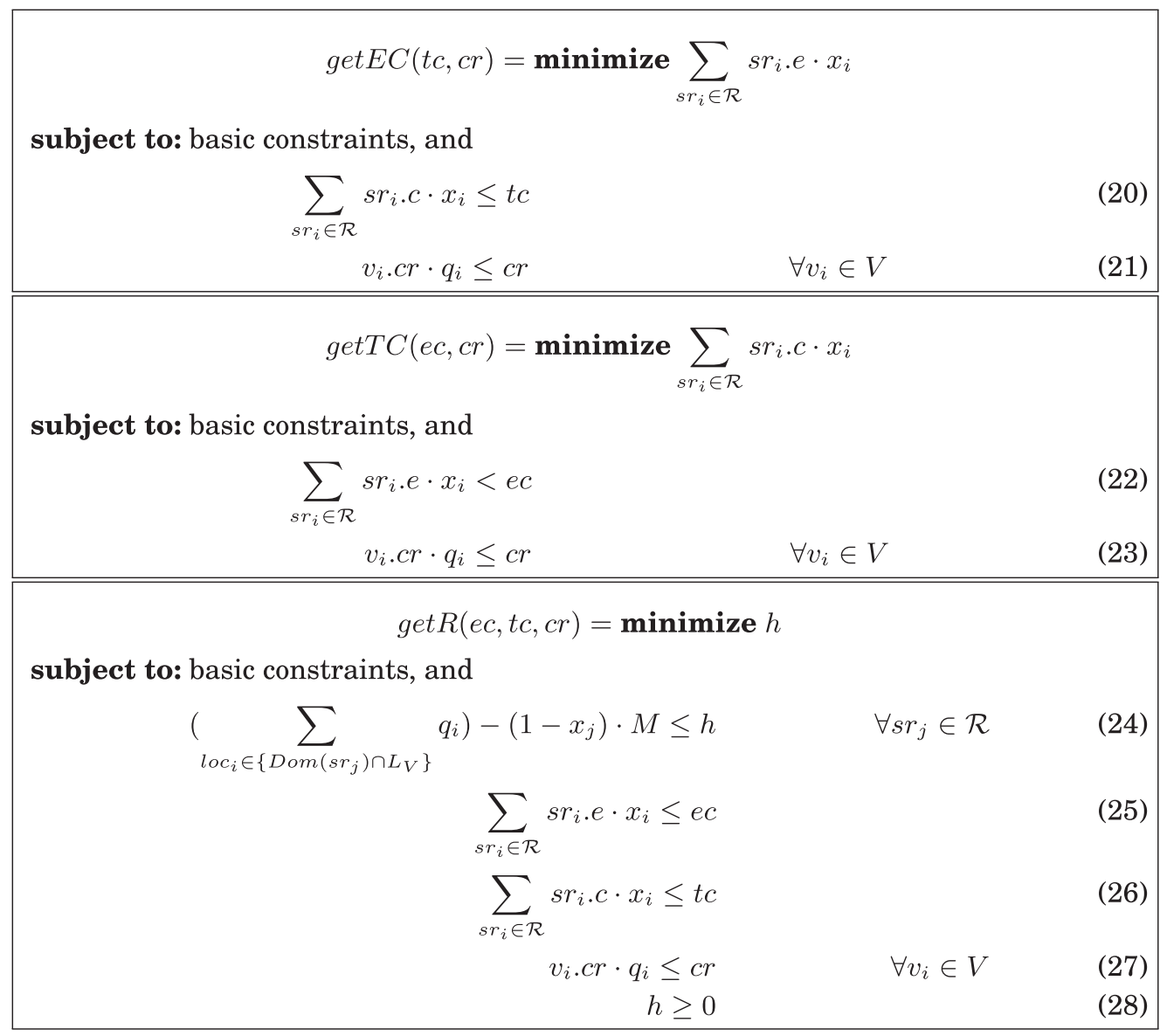

Fig. 3. Linear programs for computing the best defender strategy.

\subsection{Second Step: Best Defender Strategy}

Once we have computed the Pareto curve $P C$, we can choose the point $p^{+}=\left(e c^{+}, t c^{+}, c r^{+}\right) \in P C$ closest to our requirements of energy consumption, cost, and criticality. $p^{+}$identifies the set $A P^{+}$of different allocation plans that have an energy consumption equal to $e c^{+}$, a cost equal to $t c^{+}$, and a criticality value equal to $\mathrm{cr}^{+}$. Among all plans in $A P^{+}$, we choose the one which minimizes the risk, that is, the maximum number of unprotected nodes an attacker can exploit after compromising one security resource. To do that, we solve the optimization problem defined by Equation (2) and formalized as shown in Figure 3(bottom). The intuition behind constraint 24 is that instead of bounding the risk associated with each security resource $s r \in A P$ on the right-hand side of this constraint, we set it to an unknown value $h$ and then require the objective function to minimize $h$. Here, $M$ is a constant big enough to ensure the satisfaction of the equation for all values of $q_{i}$ and $x_{j}$ (any integer greater than $|V|$ ). Constraints 25, 26, and 27 impose that the allocation plan must be computed to have an energy consumption, a cost and a criticality value not greater than $e c, t c$, and $c r$, respectively. 


\subsection{Discussion}

Our method computes the best defender strategy based on four objectives, which are energy consumption, cost, and criticality, mutually optimized in a first phase (see Section 5.3), and risk, minimized in a second phase (see Section 5.5). We chose to split the optimization process in those two phases for three main reasons. First, a Pareto analysis with more than three competing goals may require much more time, or, alternatively, a much higher computing power to be performed. Second, a Pareto analysis involving fewer than three competing goals provides a much smaller set of solutions, thus limiting the administrator's decisional power. The reason is that the definition of dominated point becomes less restrictive when the number of dimensions decreases (see Section 5.2). The choice to optimize criticality in the first phase in place of risk is mainly a matter of convenience, since the range of criticality values is always much smaller than the range of risk values (risk $\in[0,|V|]$ ), and thus it is faster to enumerate (see Line 3 of Algorithm 1). By the way, the order in which the objectives are optimized is primarily a choice of the security officer, who might decide to employ the risk in the first phase in place of criticality because more important for the security of the network.

\section{EXAMPLES OF REAL CASE SCENARIOS}

In this section, we show how the proposed approach works for three real case IoT scenarios. The examples we address here are as follows: (1) a wireless sensor network exposed to several kinds of attacks, (2) a domestic IoT environment exposed to jamming attacks, and (3) a smart street lighting system exposed to DoS attacks.

Example 1. To make sure to counteract different kinds of attacks, the defender should secure both nodes and links. Suppose that the available security tools are an intrusion detection system (IDS), a highly sensitive transceiver (HST), ${ }^{3}$ and a tamper resistant sensor node (TRN). The defender can use tamper-resistant sensor nodes in place of normal nodes (node hardening), to prevent the attacker from having free access to node's memory, and install an IDS on (additional) sensor nodes for detecting and stopping ongoing attacks (link/node monitoring). Overall, the defender has available six types of security resources as a combination of a set of security tools and a (additional) sensor node:

(1) an IDS installed on a network node;

(2) an IDS installed on a network node equipped with a HST;

(3) a TRN to put in place of a network node;

(4) an IDS installed on a TRN to put in place of a network node;

(5) an IDS installed on an additional node to deploy in the network area;

(6) an IDS installed on an additional node equipped with a HST to deploy in the network area.

In this scenario we have that $T=\{1,2, \ldots 6\}$; for each security resource $s r$ such that $s r . t \in T^{\prime}=$ $\{1,2,3,4\}$, sr.loc $\in L_{V}$; for each security resource $s r$ such that $s r . t \in T^{\prime \prime}=\{5,6\}$, sr.loc $\in L_{A} ; \mathcal{R}=$ $T^{\prime} \times L_{V} \cup T^{\prime \prime} \times L_{A} ; T_{\alpha}=\{3,4\}, T_{\sigma}=\{1,2,4,5,6\}$ and $T_{\gamma}=\emptyset$. The defender assigns to the nodes a criticality value based on the amount of their in/out-going traffic.

Example 2. In the majority of cases, a jammer conducts radio interference on the links with her/his own device for disrupting nodes signal (Sharma and Ghose 2010). The defender thus chooses to adopt the link monitoring technique to detect the attack. The IoT devices used in this scenario are not user-programmable; thus, the defender can only adopt additional devices for security purposes (security resources of types 5 and 6 of the list of Example 1). The node's

\footnotetext{
${ }^{3}$ A HST increases the device's communication range, so it can interact with more network nodes.
} 

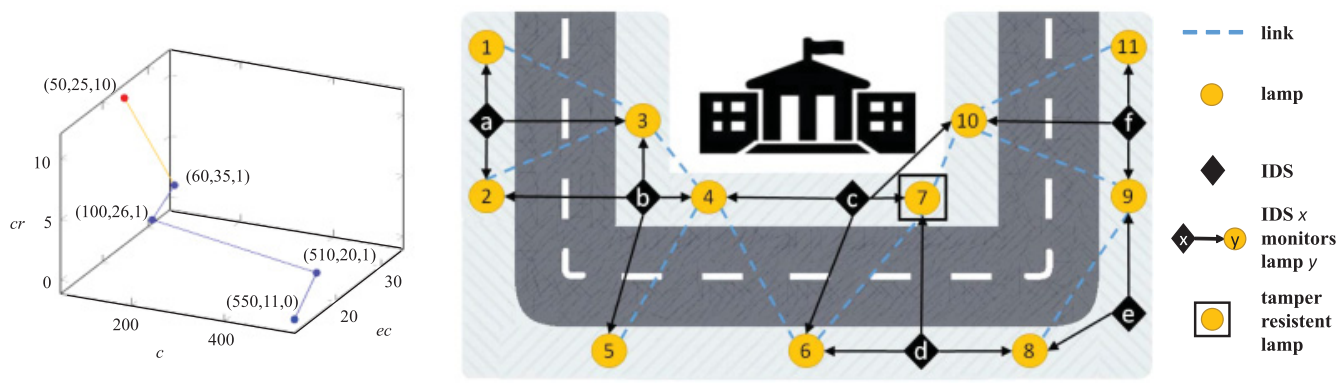

Fig. 4. Pareto curve (left) and the security reosurce allocation plans of Example 3. The Pareto points are expressend in the form $(c, e c, c r)$.

criticality is assigned customly. For this scenario we have that $T=\{5,6\}$; for each security resource $s r$, sr.loc $\in L_{A} ; \mathcal{R}=T \times L_{A} ; T_{\alpha}=T_{\gamma}=\emptyset, T_{\sigma}=T$. Notice that here the defender is only adopting the link monitoring technique, which formally means that conditions $(\alpha)$ and $(\gamma)$ are false, while conditions $(\beta)$ and $(\sigma)$ are true. In the linear programming formulation this is formalized as $T_{\alpha}=T_{\gamma}=\emptyset$ which, in Constraints 8 , forces $\sum_{s r_{j} \in \operatorname{Res}\left(v_{i} . l o c\right): s r_{j} . t \in T_{\alpha}} x_{j}$ to be 0 , and $c_{i}$ to be 1 $\left((\alpha)=\right.$ false and $(\beta)=$ true, respectively) and, in Constraints 11,16 , and 17 , forces $a_{k}$ to be 1 and $b_{k}$ to be $0((\sigma)=$ true and $(\gamma)=$ false, respectively).

In both examples, for each security resource, the values of cost, energy consumption, and communication range depend on the set of adopted tools (they can be easily determined from their data sheet) and whether an additional node is deployed.

Example 3. A DoS attack for such IoT scenario can be performed by either jamming the radio signal devices (lamps), such that they are no longer able to communicate each other, or tampering with a device in a strategic position to isolate part of the network. Suppose a system administrator willing to design the security plan before installing the lamps on the streets. (S)he can adopt two type of security resources, namely an IDS, for monitoring the links between lamps, and a tamper resistant lamp (TRL), which prevents a lamp from being compromised by an attacker. One IDS costs 10 monetary units (MU), consumes 5 energy units per hour (EUH), and can be deployed in the area delimited by the sidewalks. One TRL costs $50 \mathrm{MU}$, consumes $1 \mathrm{EUH}$, and has to be put in place of a normal lamp. The administrator has a security budget of $110 \mathrm{MU}$. Furthermore, (s)he wants to ensure a higher security level to the lamps close to the city council (lamps 3, 4, 7, and 10 of Figure 4(right)), and thus (s)he assigns a criticality value $c r=10$ to such lamps and $c r=1$ to the others. The Pareto curve computed by Algorithm 1 is shown in Figure 4(left). Two Pareto points outweigh the security budget (i.e., $(510,20,1)$ and $(550,11,0))$, and thus they are not taken into account by the administrator. The other three points represent allocation plans capable of satisfying both the security and budget constraints. At this juncture, the administrator chooses the Pareto point on which perform the second step of our optimization method. For sake of completeness, we show in Figure 4(right) the allocation plans obtained from the three Pareto points. The execution of the linear program get $R$ on the point $(50,25,10)$ returns the plan consisting of IDSs $a, b, c, e, f$. With such a plan, the nodes that would be left without security coverage in case at least one IDS stops working are two (lamps 6 and 7), thus risk $=2$. For instance, if IDS $c$ stops working (because compromised by the attacker, or for other reasons), then lamps 6 and 7 would not be covered by any other IDS. Furthermore, lamps 6 and 7 have a criticality value of 1 and 10, respectively, and thus the criticality associated to the plan is $\max (1,10)=10 . \operatorname{get} R(60,35,1)$ returns the plan 
consisting of IDSs $a, b, c, d, e, f$, that has criticality=1, because the lamps monitored by just one IDS have criticality $=1$ (lamps 1,5 , and 11 ), and risk=1, since no more than one lamp would remain unmonitored in case one IDS stops working. Finally, get $R(100,26,1)$ returns the plan consisting of IDSs $a, b, c, e, f$, and a TRL in place of lamp 7. Both criticality and risk equal 1 , because the devices monitored by just one security resource are lamps $1,5,6,8$, and 11 (all with $c r=1$ ), and no more than one lamp would remain unmonitored in case one IDS stops working.

\section{ALGORITHM SCALABILITY}

The time Algorithm 1 takes for computing the Pareto curve depends on the size of the input instance and on the number of solutions (resource allocation plans) found for that instance. ${ }^{4}$ Since we cannot know in advance the number of solutions the algorithm will find, we adopt the size of the problem as the only feature to employ in the scalability analysis together with the solving time. We define the size of the problem described by Equation (1) as the number of variables involved in the computation, which is given by the following formula:

$$
\text { size }_{P}=|\mathcal{R}|+2|V|+4|E|+\sum_{s r \in \mathcal{R}}|\operatorname{Dom}(s r)|
$$

where $|\mathcal{R}|$ is the number of $x$ variables; $2|V|$ is the number of $q$ and $c$ variables; $4|E|$ is the number of $l, a, b$, and $d$ variables; and $\sum_{s r \in \mathcal{R}}|D o m(s r)|$ is the number of $z$ variables (see Section 5.4). Notice that size $_{P}$ does depends not only on the network features $V$ and $E$ but also on Dom(sr), $\forall s r \in \mathcal{R}$, which captures the characteristics of the security resources and the number of locations where the security resources can be placed. Thus, given a set of instances different from each other for at least one of the features listed above, the algorithm will scale differently for each instance. We conducted a scalability analysis on a set of six different network scenarios that differ from each other for topology, set of security resources, ratio between locations and network nodes, and number of locations where security resources can be placed. ${ }^{5}$ Main statistics of such instances are reported in Table 1 . The set of security resources $T$ adopted for the analysis is the one described in the examples of Section 6. In particular, in the scenarios random $1-2$ and grid $1-2, T$ is the set of security resources adopted in Example 1, except for random 2 and grid2, where the communication range of the HST has been reduced by $20 \%$. In the scenarios grid3-4, $T$ is the set used in Example 2. The ratio $\mathcal{L} / V$ is 4.5 for random $1-2$ and grid1, 2.2 for grid2-3, and 1.6 for grid4. We executed this analysis on two different topologies, grid and random, as these topologies are the most commonly adopted in real-world applications. Figure 5 shows the relationship between the problem size and time taken for solving the problem for the instances of Table 1 . Notice that, given a random instance, we cannot determine in advance how long it will take to solve the instance by just looking at its size. In addition, the time does not depend on the size of the network (number of nodes); indeed, there are problems involving large-scale networks (with hundreds of nodes) that are solved in few minutes/hours, while there are instances involving less nodes but more security resources and locations that take tens of hours to be solved. Finally, the time always grows exponentially w.r.t. the size but never follows an exact exponential fashion for two main reasons: (1) The ILP solvers do not implement a simple branch and bound algorithm but use many heuristics to cut the search tree, and, consequently, many instances can be solved in a shorter solving time than that required by

\footnotetext{
${ }^{4}$ The solving time also depends on the adopted ILP solver, the implementation of the framework and the computational power of the machine used for running it, which we do not take into account for the scalability analysis because not representative of an instance.

${ }^{5}$ In this section and in Section 8 we mostly adopt grid- and random-like topologies for demonstrative purposes, because they are better suited for highlighting the behavior of the proposed algorithms. However, the proposed method is intended for working with topologies of different shapes also.
} 
Table 1. The Times Taken from Algorithm 1 for Solving the Instances of Six IoT Scenarios

\begin{tabular}{|c|c|c|c|c|c|c|c|c|c|}
\hline Scenario & Case & Size & Topology & $|\mathbf{V}|$ & $|\mathbf{E}|$ & $|\mathcal{L}|$ & $|\mathbf{T}|$ & Time (min) & Estimated time \\
\hline \multirow{6}{*}{ Random1 } & 1 & 11,420 & random & 35 & 32 & 181 & 6 & 0.43 & - \\
\hline & 2 & 14,856 & random & 51 & 51 & 221 & 6 & 1.08 & - \\
\hline & 3 & 18,052 & random & 62 & 56 & 265 & 6 & 5.30 & - \\
\hline & 4 & 22,647 & random & 70 & 83 & 313 & 6 & 19.78 & - \\
\hline & 5 & 25,922 & random & 84 & 82 & 365 & 6 & 167.52 & 133 \\
\hline & 6 & 29,926 & random & 89 & 86 & 421 & 6 & 954.33 & 989 \\
\hline \multirow{5}{*}{ Random2 } & 7 & 6,494 & random & 33 & 30 & 145 & 6 & 0.47 & - \\
\hline & 8 & 8,079 & random & 35 & 32 & 181 & 6 & 2.70 & - \\
\hline & 9 & 10,452 & random & 51 & 51 & 221 & 6 & 19.55 & - \\
\hline & 10 & 12,479 & random & 58 & 53 & 265 & 6 & 87.15 & - \\
\hline & 11 & 15,468 & random & 74 & 78 & 313 & 6 & $1,869.78$ & 1,175 \\
\hline \multirow{5}{*}{ Grid1 } & 12 & 19,102 & grid $6 \times 6$ & 36 & 60 & 169 & 6 & 0.32 & - \\
\hline & 13 & 27,148 & grid 7x7 & 49 & 84 & 225 & 6 & 0.68 & - \\
\hline & 14 & 47,464 & grid $9 \times 9$ & 81 & 144 & 361 & 6 & 8.02 & - \\
\hline & 15 & 59,734 & grid $10 \times 10$ & 100 & 180 & 441 & 6 & 18.92 & - \\
\hline & 16 & 73,412 & grid $11 \times 11$ & 121 & 220 & 529 & 6 & 435.58 & 495 \\
\hline \multirow{5}{*}{ Grid2 } & 17 & 4,818 & grid $6 \times 6$ & 36 & 60 & 85 & 6 & 0.40 & - \\
\hline & 18 & 6,756 & grid 7x7 & 49 & 84 & 113 & 6 & 2.02 & - \\
\hline & 19 & 9,022 & grid $8 \times 8$ & 64 & 112 & 145 & 6 & 12.55 & - \\
\hline & 20 & 11,616 & grid $9 \times 9$ & 81 & 144 & 181 & 6 & 80.17 & - \\
\hline & 21 & 14,538 & grid $10 \times 10$ & 100 & 180 & 221 & 6 & 625.05 & 602 \\
\hline \multirow{5}{*}{ Grid3 } & 22 & 8,958 & grid $9 \times 9$ & 81 & 144 & 181 & 2 & 0.07 & - \\
\hline & 23 & 13,794 & grid $11 x 11$ & 121 & 220 & 265 & 2 & 0.61 & - \\
\hline & 24 & 19,670 & grid $13 \times 13$ & 169 & 312 & 365 & 2 & 1.00 & - \\
\hline & 25 & 26,586 & grid $15 \times 15$ & 225 & 420 & 481 & 2 & 95.9 & - \\
\hline & 26 & 30,434 & grid $16 \times 16$ & 256 & 480 & 545 & 2 & 226.92 & 146 \\
\hline \multirow{6}{*}{ Grid4 } & 27 & 4,167 & grid $9 \times 9$ & 81 & 144 & 133 & 2 & 0.04 & - \\
\hline & 28 & 6,119 & grid $11 x 11$ & 121 & 220 & 195 & 2 & 0.17 & - \\
\hline & 29 & 8,531 & grid $13 \times 13$ & 169 & 312 & 267 & 2 & 0.40 & - \\
\hline & 30 & 12,232 & grid $15 \times 15$ & 225 & 420 & 355 & 2 & 2.24 & - \\
\hline & 31 & 14,845 & grid $17 x 17$ & 289 & 544 & 444 & 2 & 87.02 & - \\
\hline & 32 & 18,537 & grid $19 \times 19$ & 361 & 684 & 556 & 2 & 881 & 648 \\
\hline
\end{tabular}

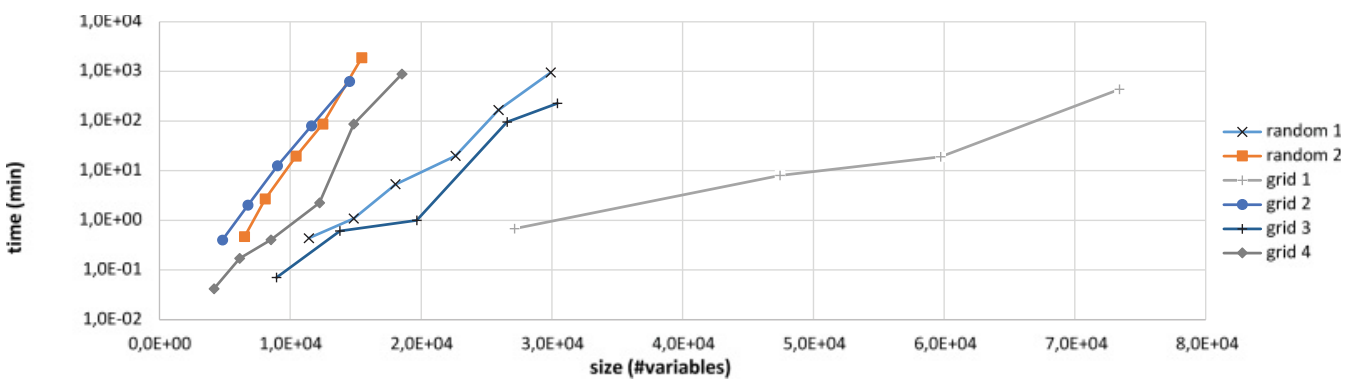

Fig. 5. How the time grows w.r.t. the problem size. 
the worst case; (2) there is no relationship between the number of solutions found by the algorithm and the size of the instance, and, thus, when the size increases the number of solutions might not, in which case the solving time does not significantly grow.

For the instances for which the optimization problem might require an unreasonable amount of time to be solved, we propose a three-step approach that computes a set of solutions within a desired time threshold. In the first step, we split the original problem in many smaller subproblems. Then we solve in parallel each sub-problem, and, finally, we merge the partial solutions. This approach computes a set of resource allocation plans that might be non-optimal due to the redundancy caused by the lack of global view during the solution of each sub-problem. The degree of redundancy is directly proportional to the number of sub-problems, and it also depends on how accurately we split the network and merge the partial solutions. In the next sections, we provide a set of heuristics that overcome such issues.

\subsection{Computing the Maximum Problem Size}

As shown in Figure 5, different instances, despite being the same size, may require different amounts of time to be solved, which sometimes may be of tens of hours. We propose to base the estimation on the size and the time taken to solve a few problems related to representative fractions of the network of interest, that is, sub-networks of different sizes that keep the ratios \#location/\#nodes and \#nodes/\#links equal to those of the whole network. More in details, given the times $t_{1}, \ldots, t_{k}$ needed to solve the problems $p_{1}, \ldots, p_{k}$ related to each sub-network, we build the set of points $T S=\{($ problem size, solving time $)\}$, and we use TS as training set for a linear regression to estimate the time needed for solving the problem related to the whole network.

We conducted such an analysis on the scenarios listed in Table 1. For each scenario, we used the problems solved in less than 2 hours as training set for the linear regression. We chose to set the threshold to 2 hours, because we think that it is a reasonable amount of time to spend for such an analysis and also because for the scenarios of Table 1 the points with solving time $<2 h$ are enough to ensure a good estimation.

Once the estimated time has been computed, we can decide whether to split the problem or not, according to the time we want to spend for computing the Pareto curve. If the estimated time is higher than our expectation, then we choose the size $h$ for which a problem with $h$ variables of such scenario is solved within a fixed time threshold $t_{P c}$ by looking at the curve identified by the points in TS. As discussed above, unfortunately, the relationship between the problem size and the time required to solve the problem might not follow an exact exponential shape, and thus an accurate estimation is difficult to achieve. By the way, we just need to know in which order of magnitude the estimated time falls to have an idea about how long the problem will take to be solved (e.g., seconds, minutes, hours, or days). Once the threshold $h$ has been chosen, we can split the network into many sub-networks such that each related sub-problem does not exceed $h$ variables. Then, solving each sub-problem in parallel, we obtain a number of partial allocation plans in an amount of time drastically smaller than that required for solving the whole problem.

\subsection{The Splitting Problem}

The splitting problem is that of dividing the optimization problem defined by Equation (1) in $p_{1}, \ldots, p_{k}$ sub-problems, such that each $p_{i}$ does not exceed $h$ variables. To this end, we use a clustering algorithm for partitioning the network area in many sub-areas, such that each associated sub-problem does not exceed $h$ variables. Since many different network topologies exist (tree, mash, sparse, grid, star, flake, etc.), in this article, we adopt a $k$-medoids-based algorithm, which always provides the best partitioning independently from the shape of the network. The are several reasons for our choice. First, it is often the case that the clusters found by a partitioning 
method are of higher quality than those produced by a hierarchical method ( $\mathrm{Ng}$ and Han 1994). Second, among the known partitioning methods ( $k$-means, fuzzy analysis, etc.) the $k$-medoids method is very robust to the presence of outliers (i.e., data points that are very far away from the rest of the data points), a feature that well fits both sparse and non-sparse topologies. Third, the (number of) clusters found by the $k$-medoids method may depend on the order in which the objects are examined, but we actually take advantage of this drawback. Since the objects are chosen randomly, we run the algorithm many times and choose the solution with the minimum number of clusters. This way, for a random topology the algorithm always finds the best partitioning among all solutions. Some methods have been proposed that pre-assign to each object a value that is the sum of the distances from the other objects and make the algorithm choose the first $k$ objects with the smallest value, to be sure to compute the best partitioning. This heuristic works well with nonsparse topologies (e.g., grid and star) but for the other cases may provide a non-minimal number of clusters. For this reason, we prefer to use a random-based algorithm that, within a certain number of runs, will identity the order of objects that provides the best partitioning whatever the shape of the network. Furthermore, our approach is fully parallelizable, because each run is independent.

In our case, the set of objects we have to divide in $k$ clusters is the set of locations $\mathcal{L}$. Since each network node is placed on a location, the clustering process will also divide the network. $k$ is not known a priori, thus at the beginning we run the algorithm with $k=2$, and we increment $k$, since all the partitions have a size at most equal to $h$. Algorithm 2 shows the pseudo code for the partitioning of the network area.

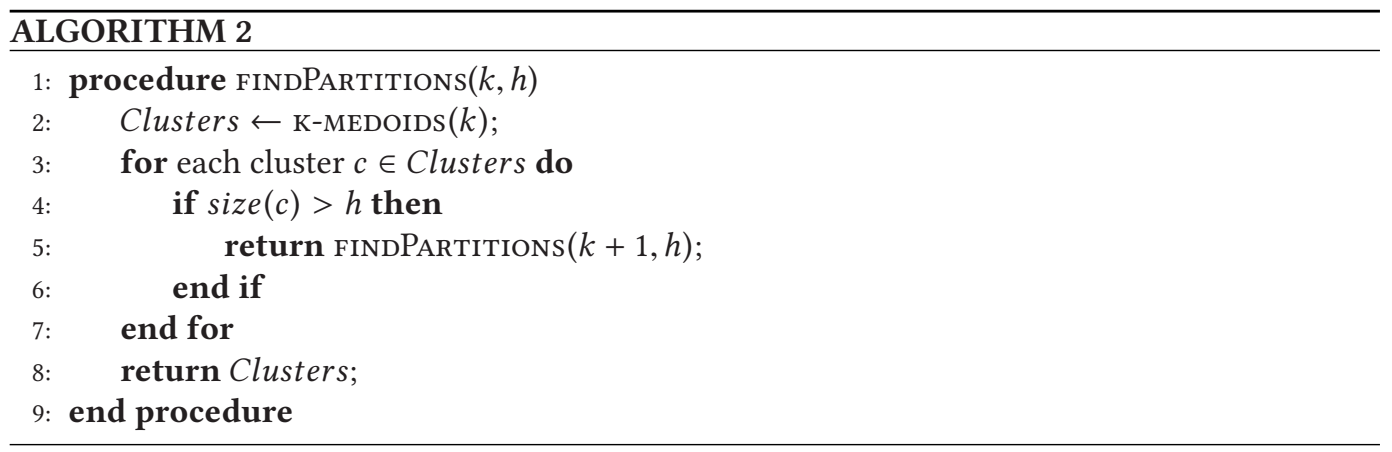

The function K-MEDOIDs $(k)$ randomly chooses $k$ objects/locations and computes the related $k$ clusters. size (c) (Line 4) is the number of variables of the problem defined by Equation (1) applied to the area covered by cluster $c$.

Once the clusters have been computed, a further step is required to complete the splitting process. As shown in the toy example of Figure 6(a), after Algorithm 2 has computed the clusters, there are edges that do not belong to any cluster (bold-dotted edges). Those edges need to be included in at least one cluster; otherwise, they would not be taken into account during the solution of each sub-problem. To this end, we expand each cluster to embed all the cut edges in at least one cluster. A simple method for effectively expanding the clusters is described by the pseudo-code of Algorithm 3. The algorithm takes as input the set of clusters computed by function FINDPARTITIONS and the set of edges $E$ of the whole network. First, it computes the set of cut edges (Line 2 ). Then it picks randomly a cut edge $\left(v_{1}, v_{2}\right)$ (Line 3 ), such that $v_{1}$ belongs to a cluster $c_{1}$ and $v_{2}$ belongs to a cluster $c_{2}$, with $c_{1} \neq c_{2}$ (Lines 4 and 5). Function EXPANDCLuster $(c, v)$ expands cluster $c$ by including $v . l o c$ and all its adjacent locations in it. Then $\left(v_{1}, v_{2}\right)$ is removed from CutEdges (Line 10), and the procedure recursively calls itself with the new set of clusters as input (Line 13), 


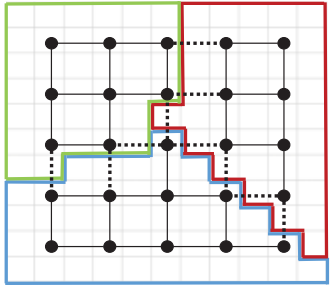

(a)

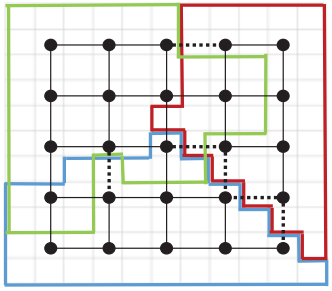

(b)

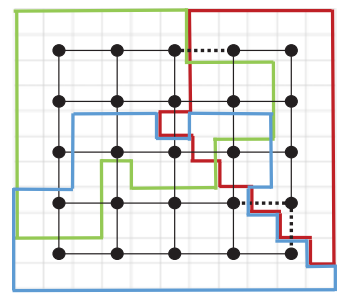

(c)

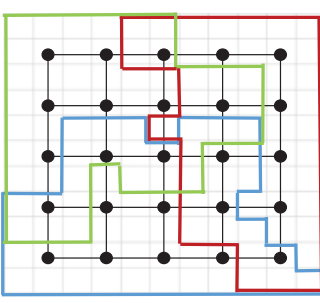

(d)

Fig. 6. Splitting process: (a) the partitions produced by the FINDPARTITION algorithm; ((b), (c), and (d)) how green/blue/red cluster embeds the cut edges after the EXPANDPARTITIONS call. The black circles represent network nodes; the squares on the background represent locations of the area in which the network is deployed.

unless there are no more cut edges, in which case the algorithm returns the set of expanded clusters (Line 12). Note that we always expand the smaller cluster (Line 6) to maintain the aspect ratio of the clusters. Figure 6 shows the partitions produced by the FINDPARTITIONS algorithm and how the expansion of each partition embeds the cut edges after each recursive call. ${ }^{6}$

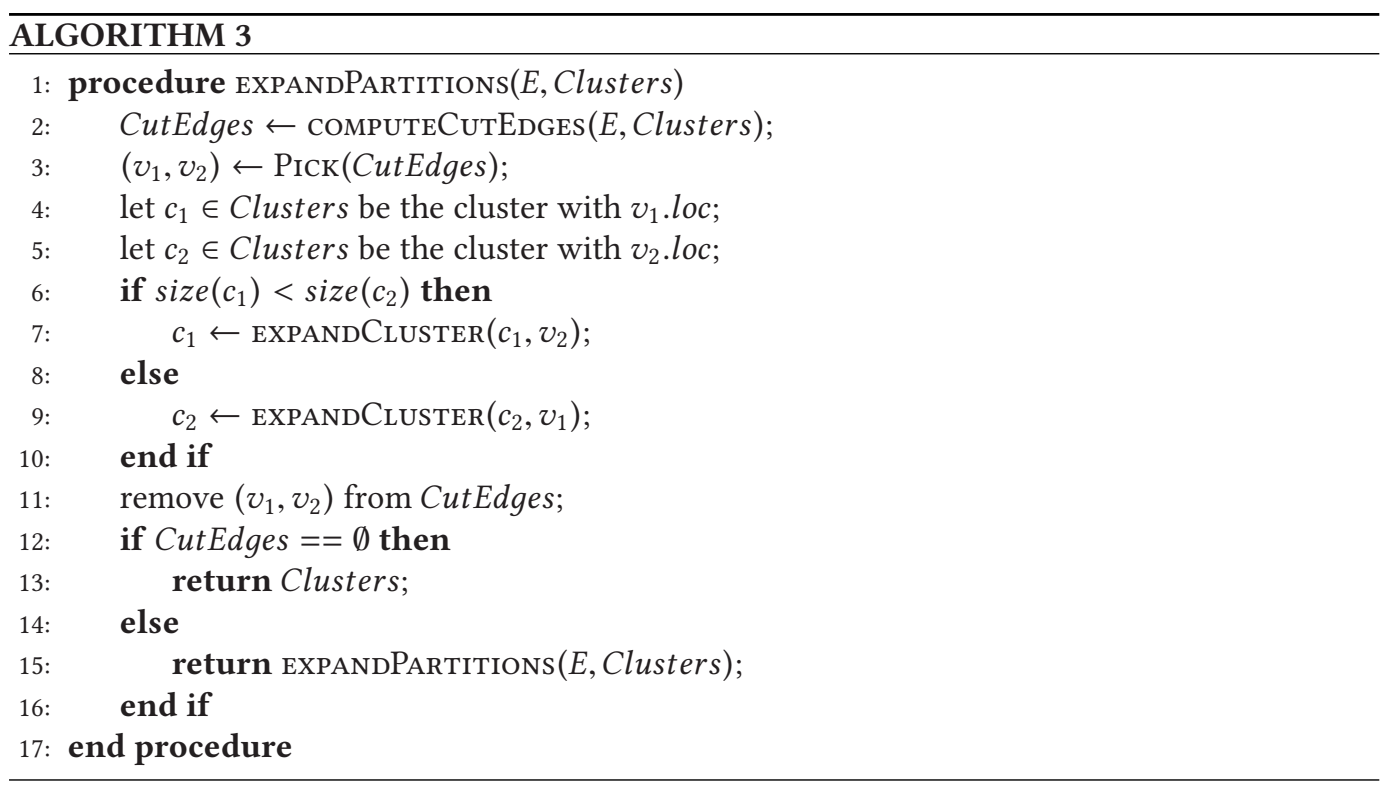

\subsection{The Merging Problem}

Once the problem has been split in $k$ smaller sub-problems, we can compute in parallel each subproblem and merge the partial solutions. This process consists of the following steps:

\footnotetext{
${ }^{6}$ Notice that after the expansion, the problems related to each cluster will have a size bigger than that chosen in the first step but not enough to represent a consistently increment of solving time.
} 
(1) for each sub-problem $p_{i}$ compute the Pareto curve $p c_{i}$;

(2) for each point (allocation plan) $(e c, c, c r) \in p c_{i}, \forall i=1 \ldots k$ compute the best defender strategy by solving the optimization problem getR(ec,c,cr) defined in Section 5.5. We obtain $S_{1} \ldots S_{k}$ sets of allocation plans $(e c, c, c r, r)$;

(3) merge sets $S_{1} \ldots S_{k}$ into set $S$;

(4) for each allocation plan in $S$ remove the redundant resources.

In steps 1 and 2, we compute a set of Pareto-optimal resource allocation plans for each of the $k$ partitions of the network area. In step 3 we merge the $k$ sets of (partial) allocation plans to obtain the set $S$ of plans that cover the whole network area. Due to the lack of global view during the computation of the $k$ sub-problems, and to the partial overlapping of the $k$ clusters, the set $S$ contains redundant allocation plans, that is, plans consisting of security resources whose domains excessively overlap because originally belonging to different clusters. In step 4 , we remove the redundant resources from each plan in $S$. Algorithm 4 describe how we merge the sets $S_{1} \ldots S_{k}$ in set $S$.

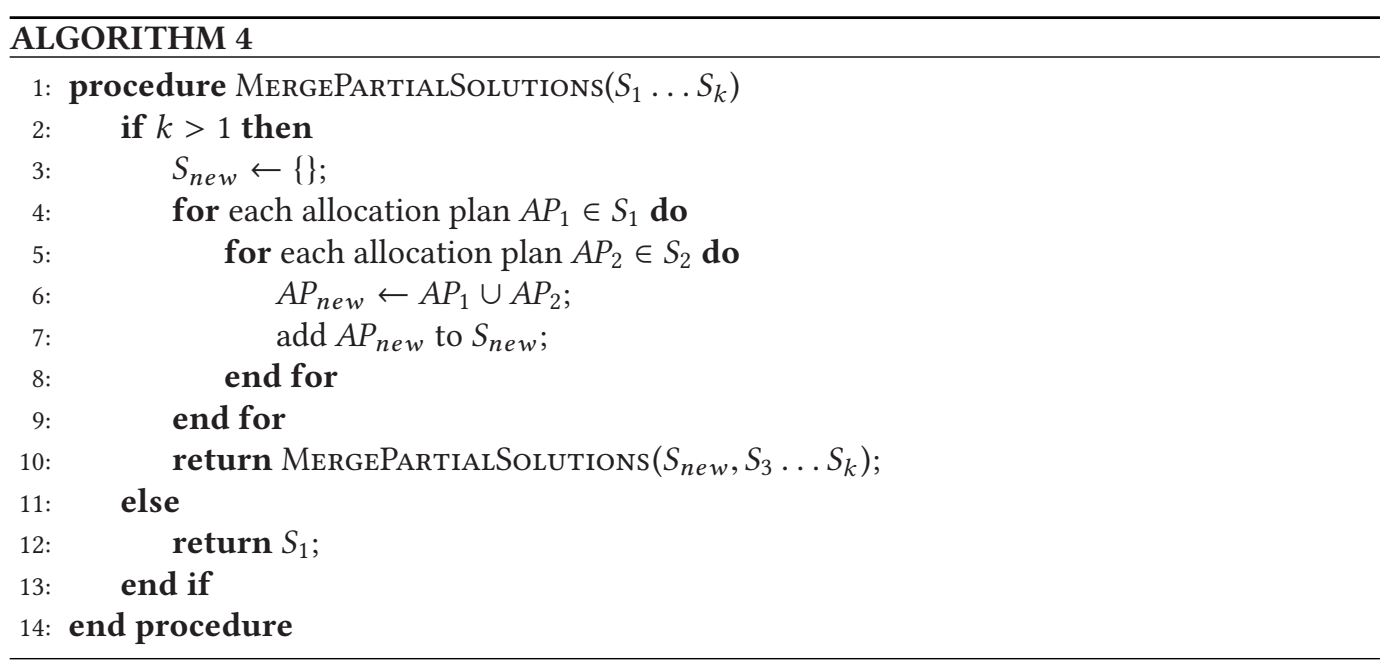

Algorithm 4 is a recursive function that takes as input the list $S_{1} \ldots S_{k}$ of sets computed in step 2, merges the allocation plans in $S_{1}$ with the allocation plans in $S_{2}$ (Lines 4-6), and adds the results in $S_{\text {new }}$ (Line 7). Then $S_{\text {new }}$ is passed as first element of the list in place of $S_{1}$ and $S_{2}$ for the recursive call of Line 10. This way the algorithm reduces the size of the list until it contains just one set, which is returned as the set of the merged allocation plans (Line 12).

Algorithm 5 removes the redundant resources from $S$. The algorithm follows the same approach of Algorithm 1, that is, for each criticality value (Line 5) selects the security resources that minimize the total energy consumption and the total cost. In particular, for each allocation plan $A P \in S$ the algorithm removes one by one the redundant resources, that is, resources whose domain is covered by the other resources of $A P$ and whose removal does not increase the current risk (Line 8). Once the redundant resources have been removed, $A P$ is added to $X$ (Line 12). Finally, the algorithm selects the non-dominated allocation plans of $X$ (Line 15).

\subsection{Complexity Analysis}

Considered that Algorithm 1 has an exponential component in its execution time, due to the number of variables of the integer linear formulation (multiplied by the number of Pareto points), in this 


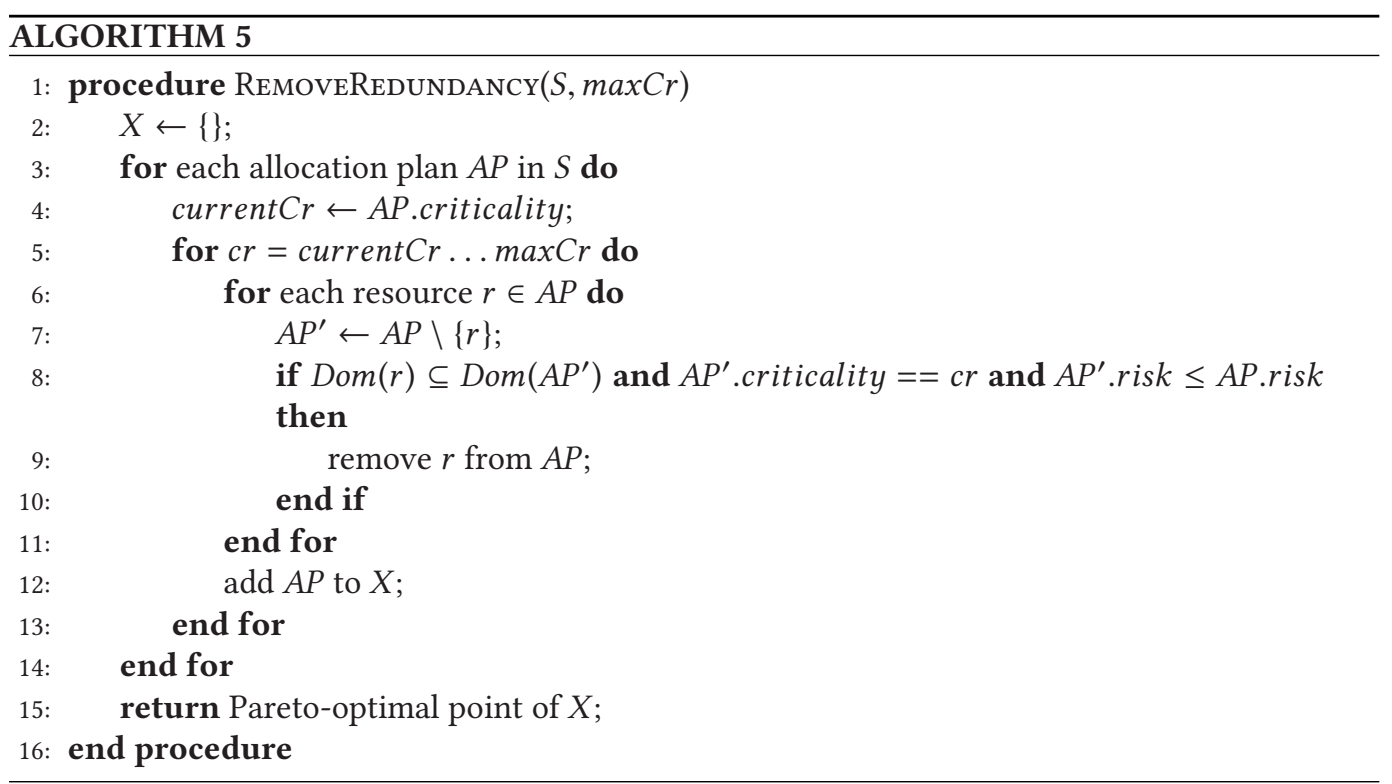

section we limit our analysis in discussing which of our algorithms are polynomial or exponential and what is the size of the exponent.

Algorithm 1 solves two ILPs for each Pareto point that cost exponential time in the number of the ILP variables given by Equation (4), thus $O\left(\exp \left(p o l y\left(\operatorname{size}_{P}\right)\right)\right)$. Moreover, in the worst case, the Pareto curve consists of a number of points (very rare cases) that are exponential in the number of bit (precision) used for representing power consumption, criticality, and cost, and thus $O(\exp (3 *$ precision $))$. In total, the computational complexity of Algorithm 1 is $O(\exp (3 *$ precision $) * \exp \left(\right.$ poly $\left(\right.$ size $\left.\left.\left._{P}\right)\right)\right)$.

Algorithms 2 and 3 are polynomial in the number of clusters $k$ and in the number of locations $|\mathcal{L}|$. The merging procedure (Section 7.3) is composed of four steps. In the first one, for each cluster we execute Algorithm 1 with an input instance of size at most $h$ (the maximum size of each partition), thus the complexity is $O(k * \exp (3 *$ precision $) * \exp (\operatorname{poly}(h)))$. In the second step, we solve the ILP getR, which has the same complexity of the first step. In the third step, we execute Algorithm 4, which costs $O(k * \exp (3 *$ precision $))$, because it merges $k$ Pareto curves of at most $\exp (3 *$ precision $)$ Pareto points. In the last step, we execute Algortihm 5 , which costs $O(\exp (3 *$ precision $))$, since the global allocation plan computed in the previous step has a maximum (theoretical) number of point of $\exp (3 *$ precision $)$.

To conclude, the dominant component among the computational complexities of the four algorithms is $O(k * \exp (3 *$ precision $) * \exp ($ poly $(h)))$, which is much better than $O(\exp (3 *$

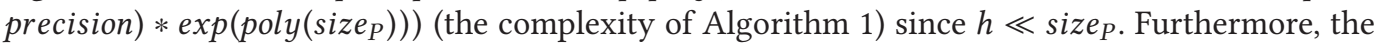
real execution times are way much smaller than those of the worst cases considered in this analysis, as demonstrated by the experiments in Section 9 .

\section{EXPERIMENTAL RESULTS}

In this section, we present the experimental results of our proposed framework when applied to the attack scenario described in Example 1 of Section 6. The goal is to demonstrate the importance of accounting for effectiveness metrics in the design process of a security infrastructure, besides 


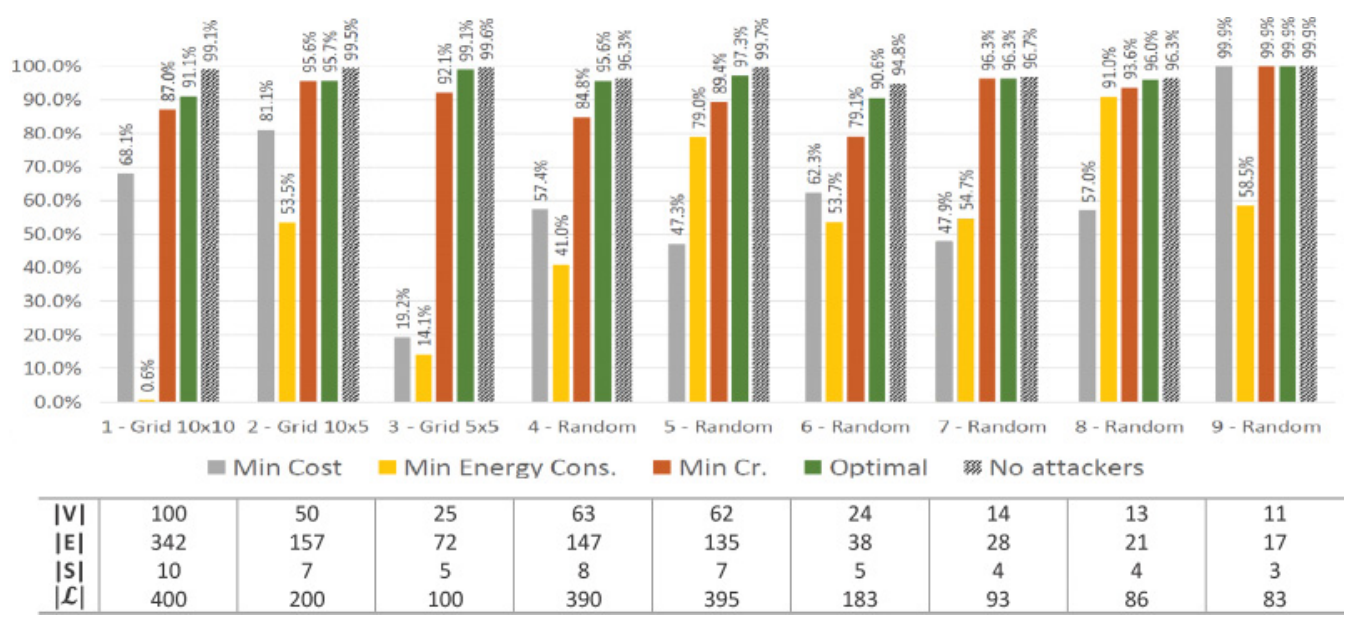

Fig. 7. Performance of the strategies for each of the topologies used in the experiments. Even in an attack-free simulation, the packet delovery rate (PDR) is never $100 \%$ due to the packet drop caused by natural physical phenomena and network operations. $\mathbf{V}$ is the set of nodes, $\mathbf{E}$ is the set of edges, $\mathbf{S}$ is the set of source nodes, and $\mathcal{L}$ is the set of locations.

efficiency. We show how the effectiveness of the best strategy is higher compared to any other strategy. We applied our technique to nine different network topologies, whose main characteristics are reported in Figure 7. For each topology, we computed four strategies: (i) the one that entails the minimum cost $A P_{c}$; (ii) the one that entails the minimum energy consumption $A P_{e c}$; (iii) the one that provides a reasonable balance among cost, energy consumption, and criticality $\left(c^{*}, e c^{*}, c r^{*}\right)$, namely $A P_{c r}$; and (iv) the best strategy $A P^{*}$ (the one that entails the minimum risk, given $\left.\left(c^{*}, e c^{*}, c r^{*}\right)\right)$. We simulated a selective forwarding attack and measured the packet delivery rate (PDR) of the network for the four strategies.

\subsection{Settings}

We implemented our framework in Java and used IBM ILOG CPLEX 12.5 (ILOG 2011) to solve the ILPs. All computations were run on an Intel Core i7-5600U CPU clocked at $2.59 \mathrm{GHz}$, running Windows 8.1 64bit, with 2GB RAM available for each experiment. To evaluate the effectiveness of the different allocation plans, we implemented the topologies in TinyOS (Levis et al. 2005) and carried out 10 independent simulations of 500s each in the TOSSIM simulator (Levis et al. 2003). We computed nodes criticality as integer values, ranging from $\min C R=1$ to $\max C R=\lceil\sqrt{|V|\rceil}$. Its computation was based on the amount of data packets $D P$ passing through a node during an attack-free simulation of 500s. We normalized the $D P$ value of each node according to a scale from $\min C R$ to $\max C R$. Then, we assigned to source nodes the maximum value plus one to assign to these nodes the maximum degree of security. The set of security resources $\mathcal{R}$ is as described in Section 6. We assigned a value for cost $c$, average energy consumption $e c$, and communication range $d$, as follows:

- network node: $c_{n}=2, e c_{n}=2, d_{n}=2$.

-IDS: $c_{I D S}=1, e c_{I D S}=2$;

-TRN: $c_{T R N}=7, e c_{T R N}=3$;

$-\mathrm{HST}: c_{H S T}=5, e c_{H S T}=3, d_{H S T}=5$. 
The values above were assigned to reflect real relative differences of cost, energy consumption, and communication range between nodes. Overall, we believe these values to be realistic for our experimental purposes.

\subsection{Results Analysis}

We simulated a selective forwarding attack (Sharma and Ghose 2010) (with 50\% probability of dropping packets) for 500s, over the nodes no longer protected when the security resource that maximizes the risk is removed from the defense strategy. Figure 7 shows the main statistics a comparison of the performances for each set of strategies, together with the PDR of an attack-free scenario. First, we note that the most efficient strategies $A P_{c}$ and $A P_{e c}$ are not the most effective; in fact, due to the higher value of $c r$ and $r$, they provide a lower PDR with respect to $A P_{c r}$ and $A P^{*}$, that is, the strategies computed by taking into account effectiveness metrics, too. Furthermore, we observe that the PDR is inversely proportional to the risk and criticality values, since the more relevant and numerous the unprotected nodes are, the more the lost data packets are. In more detail, in Case $1 A P_{c r}$ and $A P^{*}$ provide the same criticality, but $A P^{*}$ entails a lower risk and, as a consequence, a higher PDR. In Case 2, we observe that $A P_{c}$ and $A P_{c r}$ provide the same risk, but $A P_{c}$ entails a lower PDR, since its criticality is higher. In Case 7, we have that $A P_{c r}$ and $A P^{*}$ are exactly the same, because the set of strategies with $c r=1$ has cardinality 1 , that is, only one strategy exists and, consequently, it is also the best one. In Case 9, the cheapest strategy $A P_{c}$ is also the best strategy for $c r=1$. Furthermore, notice that we always chose an $A P_{c r}$ with criticality greater than 0 , which implies a risk greater than 0 , and then we are able to provide a more meaningful comparison of the strategies. If we had chosen $A P_{c r}$ always with $c r=0$, then the risk would have also been 0 , and $A P^{*}$ would have always been exactly the same as the risk of $A P_{c r}$. Overall, we note that the quality of a strategy usually increases with the introduction of effectiveness metrics in the computation process. Hence, for all the cases (apart case 9), $A P_{c r}$ results in a higher PDR than $A P_{c}$ and $A P_{e c}$, and the best strategy $A P^{*}$-the one computed by taking also into account the risk besides efficiency and criticality-is even better than $A P_{c r}$. We conclude that our approach provides the best defender strategy, which provides the higher security level at the minumum cost and energy.

\section{EVALUATION OF THE THREE-STEP APPROACH FOR LARGE INSTANCES}

In this section we evaluate the three-steps approach proposed for solving instances that require a large amount of time. We base the evaluation on the time taken to compute the solutions (resource allocation plans) and on their quality in terms of efficiency and effectiveness. Figures 8 and 9 show the results of such evaluation conducted on the largest networks of five of the eight scenarios listed in Table 1 (i.e., the cases that involve the highest solving time). For each scenario, we compared the set $S$ of solutions obtained by considering the problem as a whole, with the set $S^{\prime}$ of solutions computed with the three-steps approach. Furthermore, we repeated such analysis with different number of clusters to show how the solutions quality varies. In all cases, the three-steps approach consistently reduces the solving time but at the expense of efficiency. The resource allocation plans are non-optimal due to redundancy introduced by the partial overlaps of the clusters and the lack of global view during the solution of the sub-problems. In fact, the average cost and energy consumption of the solutions in $S^{\prime}$ are higher then those of $S$. However, we can notice also that the average risk is always lower. In fact, if on the one hand redundancy penalizes the efficiency, then, on the other, it increases the effectiveness, since a higher number of security resources (redundancy) decreases the probability of successful attacks. Moreover, we can observe that the larger the number of clusters, the higher the efficiency loss, which in some cases is consistently low w.r.t. the optimal solution. However, we can appreciate how, for each scenario, the cases with an 


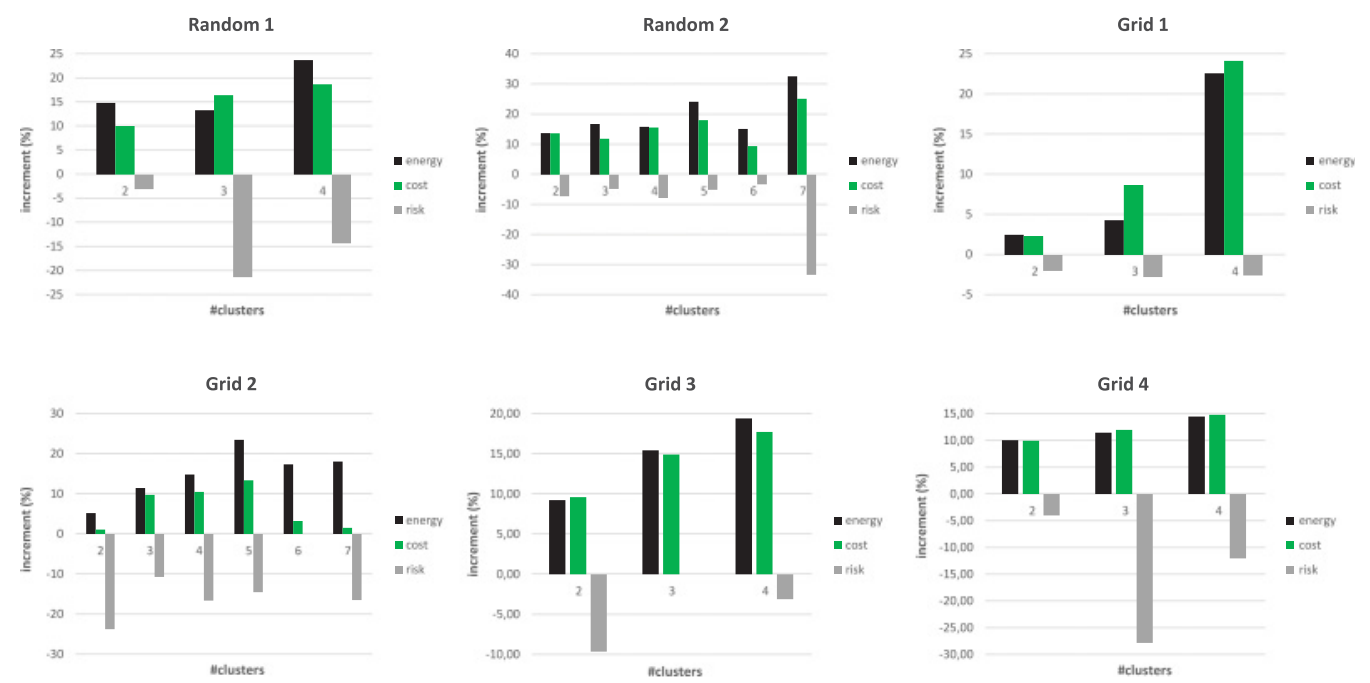

Fig. 8. Increment of energy, cost, and risk in the solutions computed by the three-steps approach w.r.t. the optimal solutions.
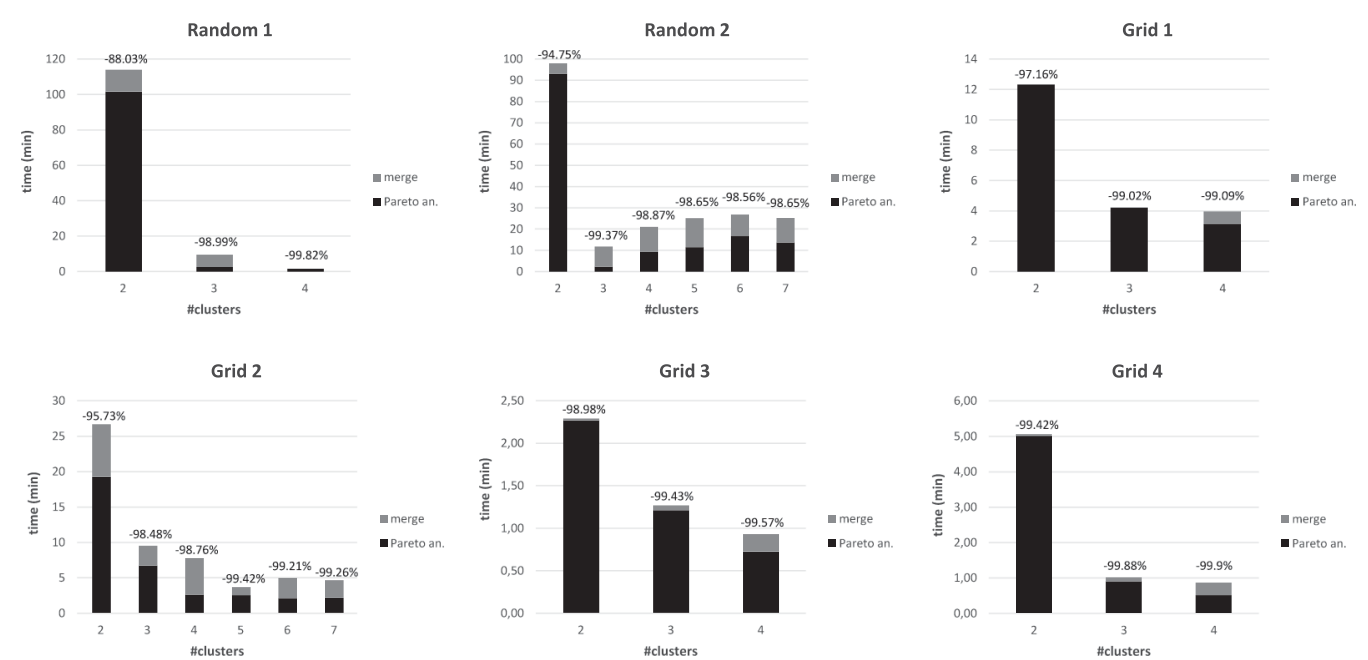

Fig. 9. Time spent by the three-steps approach for computing a set of solutions for the largest networks of five of the eight scenarios listed in Table 1. The percentages indicate the time saved w.r.t. those of Table 1.

affordable increment of cost and energy consumption $(\leq 15 \%)$ provide a reasonable solving time w.r.t. the time required to solve the same problem without the preliminary clustering (never less than $88 \%$ of time saved). ${ }^{7}$ It is also interesting to see that when increasing the number of clusters, the results of energy, cost, and risk do not change monotonically in all the topologies. This can be

\footnotetext{
${ }^{7}$ Our analysis does not take into account the criticality as an evaluation metric, because the percentage increase of average criticality of $S^{\prime}$ w.r.t. $S$ would mostly depend on the range of criticality values. Indeed, with a small range, for example, $[1,3]$, the percentage increase might go up to $\pm 300 \%$, while with a larger range, for example, $[1,15]$, the increase would be consistently smaller. By the way, the criticality value will always decrease due to the introduced redundancy.
} 
explained by the random behavior of the $k$-medoids method, chosen as clustering method in Algorithm 2, and to the random order in which redundant resources are removed from an allocation plan (see Algorithm 5). To address such drawbacks, due to the random behavior of the three-steps approach, one can carry out experiments with different numbers of clusters, as they only take few minutes, and, finally, choose the one that provides the best results.

\section{FURTHER USES OF THE PROPOSED APPROACH}

The problem of optimally distribute a set of resources has already been largely considered in many research domains. IoT is today a relatively new research field, and as far as we know, we are the first to provide a formalization for solving this problem when applied to an IoT security scenario. We do not take into account any real-world security resource and IoT device feature; rather, we focus on the designing of a framework that gives the guidelines for optimally placing security resources in IoT scenarios. Thus, our formalism does not refer to any specific situation, but it is designed to be a general approach easy to adapt to different cases. As a matter of fact, the metrics we adopted to describe the concepts of efficiency and effectiveness of a security infrastructure are general enough to be exploited for many IoT networks. The node's criticality can assume different meanings depending on the security needs (see Section 5.1); the resource's cost can be referred to any source of expense: the budget to spend for buying security resources and the budget needed for their maintenance and for their installation. The algorithm that computes the Pareto frontier can be easily converted from a three-objective to a bi-objective optimization algorithm, or from a two-phase to an $n$-phase algorithm, according to the number of objectives a security officer wants to optimize. There are cases in which one or more of the four objectives do not matter for a particular IoT scenario. For instance, in a home environment is often the case that energy consumption is not a metric of interest, since in such small areas the electrical power is always available. Or else, in a one-hop WSN where all nodes perform the same sensing task, there is no criticality. Therefore the second phase is not required, as the risk can be evaluated concurrently with the other two objectives, or, alternatively, the first phase can be converted in a two-objective optimization problem. The cost might be not relevant for small-scale networks and can thus be discarded from the analysis. Furthermore, to restrict the set of solutions of interest, and thus to speed up the optimization process, one can impose bounds on the objectives values. For all these cases it is easy to adapt our framework.

For the sake of simplicity, we chose to not differentiate security resources based on their level of effectiveness, but rather we assume them to be equally good. It is surely a limitation of our approach; however, the framework can be easily enriched with further features. Indeed, the security administrator can differentiate resources based on their effectiveness by adding a new feature, for example, weight, to the already existing ones $\langle c, e, t, l o c\rangle$ (see Section 3.1). This way, the administrator can maximize the total weight as another objective to make the security infrastructure more effective.

A further use of the proposed method is in the performance assessment of security tools. For a given attack, different detection/prevention techniques might exist for which efficiency and effectiveness depend on some feature of the network of interest, such as topology, mobility, routing protocol, and so on. A security administrator may want to perform a simulation to determine which IDS/IPS provides the best performance in his/her specific case. Our framework can also be used in research as an evaluation tool for the comparison of new security techniques with existing ones.

\section{RELATED WORKS}

The problem of finding efficient security solutions with the help of game theory and Pareto analysis has been extensively considered for computer networks (Altman et al. 2009; Cheng and Zhuang 
2009; Han et al. 2009; Dewri et al. 2012; Poolsappasit et al. 2012; Serra et al. 2015; Zhu et al. 2012). In Serra et al. (2015) the interaction between attacker and defender is modeled as a Stackelberg game, and Pareto analysis is used to compute the best tradeoff between the cost of patching vulnerabilities and the cost due to the deactivation of vulnerable products within an enterprise system. In Poolsappasit et al. (2012), the authors consider the problem of finding plans for patching vulnerabilities, that are tradeoffs between cost and risk, by using the Pareto analysis. In Dewri et al. (2012), the authors define a problem involving a game-theory-based solution, but they do not mix it with the Pareto analysis and assume that the attacker does not know the strategy of the defender (we instead assume this is possible). The approach in Altman et al. (2009) uses game theory to study jamming attacks, while the approach in Han et al. (2009) uses Stackelberg games to model the interaction between defender and eavesdroppers. We like also to mention Zhu et al. (2012) and Cheng and Zhuang (2009) as typical examples of game theory and Pareto analysis applied to IoT scenarios. The approach in Zhu et al. (2012) investigates sensor networks in which an attacker can physically capture, replicate the nodes, and deploy sensors into a network and then proceed to take over the network. A multi-player game is formalized to model the non-cooperative strategic behavior between the attackers and the network. The approach in Cheng and Zhuang (2009) is based on a node clustering algorithm, with effective tax-based sub-carrier allocation tailored for wireless mesh networks with QoS support. Here, Pareto analysis is used for the optimal resource management. Other work focuses on security in the physical layer from eavesdropping and jamming attacks. In such previous approaches, players include attackers, non malicious users (that use the physical layer), and the layer itself (with its access control policy). These games are largely based on performance indexes of the physical layer, and the main goal is to optimize these performance indexes.

Solutions proposed in the context of IoT have focused on efficiency, due to the small "size" of network components, in terms of CPU, memory, and energy budget. Zhou and Chao (2011) propose a media-aware security framework for facilitating various applications in IoT, and they provide a design rule and strategy to achieve a good tradeoff between system's flexibility and efficiency. Raza et al. (2014) propose an IPsec extension of 6LoWPAN and show that IPsec is a feasible option for securing the IoT in terms of packet size, energy consumption, memory usage, and processing time. In Chigan et al. (2005), the authors propose a resource-aware self-adaptive network security provisioning scheme for the resource constraint Mobile Ad-hoc Networks (MANET) to avoid security provisioning Denial of Service (SPDoS) attack. In this work, efficiency and effectiveness are modeled as two indexes, performance index (PI) and security index (SE), respectively. SI quantitatively reflects the security contribution of a secure protocol (set) to a MANET system: higher SI indicates a protocol (set) is more resistant to various attackers. PI quantitatively reflects network performance perspectives of a secure protocol (set): It is based on the QoS parameters (e.g., throughput, latency, jitter, power consumption, etc.); a higher PI indicates a secure protocol (set) of lower performance cost.

Many algorithms are available for computing the Pareto frontier; one of the most used is NSGAII (Deb et al. 2000). NSGA-II is an evolutionary genetic algorithm able to find an approximation of the Pareto frontier for multi-objective optimization problems. The main difference between NSGAII and others evolutionary genetic algorithms is the selection phase, which in NSGA-II does not have a unique fitness function but one for each object function. During the selection phase, the selected points are only the non-dominated ones. This approach works very well when the optimization problem is unconstrained. When some constraints exist, the selection phase will remove all the points that do not satisfy the constraints. This kind of naive solution takes a lot of time to reach a good approximation of the Pareto frontier. Since our problem is a constrained multioptimization problem, we prefer to use an approach with normal constraints that is more efficient 
and effective. NSGA-II is used by Dewri et al. (2008) as an optimization approach for minimizing the residual damage and the cost of security provisioning. They propose the use of workflow profiles to capture the contexts in which a communication channel is used in a pervasive environment. This is used to minimize the cost that the underlying business entity will have to incur to keep the workflow secure and running. Unlike us, they do not provide a formalization of the linear programs.

Many approaches have been proposed for computing the criticality of graph nodes. Recent approaches target the distributed evaluation and placement of the nodes most critical to network robustness, thus assessing node centrality in a distributed way (Dinh et al. 2010). Marsden (2002) shows empirical evidence that localized centrality measures calculated for one-hop radius neighborhood are highly correlated to the global centrality measure. Kermarrec et al. (2011) propose a new centrality measure, called second-order centrality, defined in terms of the standard deviation of the time between visits of a perpetual random walk to each node. As alternative, simulation tools (Levis et al. 2003; Girod et al. 2004; Varga et al. 2001) can help in understanding the relevance of a node for the correct function of the network. For instance, in a wireless sensor network scenario, where sources nodes collect data from the environment and send them to a sink through several edge nodes, a simulation can help understanding what are the most critical edge nodes, based on the amount of traffic involving them. Whereas the IoT system is already deployed, one can easily query network nodes about performances, in/out-coming traffic, and so on.

To the best of our knowledge, the problem of finding the optimal security resource allocation plan for IoT networks has never been investigated, except in our recent articles (Rullo et al. 2017a, 2017b), which provide a solution for mobile IoT networks. The current article extends our previous works (Rullo et al. 2017a) as follows: (i) it presents several methods for computing nodes criticality (Section 5.1); (ii) it provides the set of basic constraints that capture the dependencies between the variables used in the linear programs (Section 5.4); (iii) it provides two real case scenarios as examples of how to implement the proposed method (Section 6); (iv) it introduces a heuristic for computing the best allocation plan for large scale networks (Section 7); and (v) it provides a discussion of further uses of the proposed approach (Section 10). Past work on IoT security focuses on protection mechanisms against specific attacks (Tumrongwittayapak and Varakulsiripunth 2009; Altman et al. 2009; Zhu et al. 2010); investigates ISO/OSI layer-related security problems (Jinwala et al. 2012); or proposes architectures for intrusion detection, attack prevention, or recovery systems (Raza et al. 2013; Roman et al. 2011). To assure the correct function of IoT networks even under attack, one may need to deploy many such security mechanisms. Therefore, a formal methodology for allocating such mechanisms is needed. To this end, this article proposes a method capable to work with all the different security techniques adopted by the systems mentioned above.

\section{CONCLUSION}

In this article, we develop a game-theoretic model to answer the following question: In an IoT scenario, given a set of security resources and a set of attacks to protect against, which resources should a security administrator choose, and how should (s)he allocate them in the network to ensure protection with the minimum cost, the minimum energy consumption, and a certain degree of robustness against attacks? To answer this question, we provide a method for computing the best defender strategy, that gives one the possibility of choosing the resource allocation plan that best fits efficiency and effectiveness requirements. This leads to a problem involving Pareto optimization, because efficiency and effectiveness are mutually competing goals. We show how to formulate the defender's problem as a linear optimization problem and suggest a number of measures to formalize efficiency and effectiveness aspects of the defender's strategy. We also provide a heuristic for overcoming the scalability issues we might incur when dealing with particular instances. Our 
experimental results prove that our method provides the best defender strategy compared to other strategies that do not take into account (all) effectiveness measures, but efficiency only. We also proved that for large instances our three-steps approach provides solutions with low overhead w.r.t. the optimal ones and, due to the use of parallel computing techniques, in an amount of time equivalent to that needed for small instances.

\section{REFERENCES}

Eitan Altman, Konstantin Avrachenkov, and Andrey Gamaev. 2009. Jamming in wireless networks: The case of several jammers. In Proceedings of the 1st ICST International Conference on Game Theory for Networks.

Luigi Atzori, Antonio Iera, and Giacomo Morabito. 2010. The Internet of Things: A survey. Comput. Netw. 54, 15 (2010), 2787-2805.

Ferdinand Brasser, Brahim El Mahjoub, Ahmad-Reza Sadeghi, Christian Wachsmann, and Patrick Koeberl. 2015. TyTAN: Tiny trust anchor for tiny devices. In Proceedings of the Design Automation Conference (DAC'15). IEEE, 1-6.

Srdjan Čapkun, Levente Buttyán, and Jean-Pierre Hubaux. 2003. SECTOR: Secure tracking of node encounters in multi-hop wireless networks. In Proceedings of the 1st ACM Workshop on Security of Ad Hoc and Sensor Networks.

Ho Ting Cheng and Weihua Zhuang. 2009. Pareto optimal resource management for wireless mesh networks with QoS assurance: Joint node clustering and subcarrier allocation. IEEE Transactions on Wireless Communications 8, 3 (2009), 1573-1583.

Chunxiao Chigan, Leiyuan Li, and Yinghua Ye. 2005. Resource-aware self-adaptive security provisioning in mobile ad hoc networks. In Proceedings of the IEEE Wireless Communications and Networking Conference.

Kalyanmoy Deb, Amrit Pratap, Sameer Agarwal, and T. Meyarivan. 2000. A fast elitist multi-objective genetic algorithm: NSGA-II. IEEE Trans. Evol. Comput. 6 (2000), 182-197.

Rinku Dewri, Indrajit Ray, Nayot Poolsappasit, and Darrell Whitley. 2012. Optimal security hardening on attack tree models of networks: A cost-benefit analysis. In International fournal of Information Security 11, 3 (2012), 167-188.

Rinku Dewri, Indrakshi Ray, Indrajit Ray, and Darrell Whitley. 2008. Security provisioning in pervasive environments using multi-objective optimization. In Proceedings of the European Symposium on Research in Computer Security (ESORICS'08).

Thang N. Dinh, Ying Xuan, My T. Thai, EK Park, and Taieb Znati. 2010. On approximation of new optimization methods for assessing network vulnerability. In Proceedings of the IEEE International Conference on Computer Communications (INFOCOM'10).

Laurent Eschenauer and Virgil D. Gligor. 2002. A key-management scheme for distributed sensor networks. In Proceedings of the 9th ACM Conference on Computer and Communications Security. ACM, 41-47.

Lewis Girod, Jeremy Elson, Alberto Cerpa, Thanos Stathopoulos, Nithya Ramanathan, and Deborah Estrin. 2004. EmStar: A software environment for developing and deploying wireless sensor networks. In Proceedings of the USENIX Annual Technical Conference (USENIX'04).

Lal C Godara. 1997. Application of antenna arrays to mobile communications. II. Beam-forming and direction-of-arrival considerations. Proc. IEEE 85, 8 (1997), 1195-1245.

Zhu Han, Ninoslav Marina, Mérouane Debbah, and Are Hjørungnes. 2009. Physical layer security game: How to date a girl with her boyfriend on the same table. In Proc. of the 1st ICST International Conference on Game Theory for Networks.

Yih-Chun Hu, Adrian Perrig, and David B. Johnson. 2003. Packet leashes: A defense against wormhole attacks in wireless networks. In Proceedings of the IEEE International Conference on Computer Communications (INFOCOM'03). IEEE.

Yi-an Huang and Wenke Lee. 2003. A cooperative intrusion detection system for Ad Hoc networks. In Proceedings of the 1st ACM Workshop on Security of Ad Hoc and Sensor Networks (SASN'03). ACM, New York, NY, 135-147. DOI: http://dx.doi.org/10.1145/986858.986877

Jonathan Hui, David Culler, and Samita Chakrabarti. 2009. 6LoWPAN: Incorporating IEEE 802.15. 4 into the IP architecture. IPSO Alliance White Paper 3 (2009).

IEEEE. 2007. IEEE 802.15 WPAN Task Group 4 (TG4). Retrieved from http://www.ieee802.org/15/pub/TG4.html.

IBM ILOG. 2011. CPLEX 12.5. (2011)

Devesh Jinwala, Dhiren Patel, and Kankar Dasgupta. 2012. FlexiSec: A configurable link layer security architecture for wireless sensor networks. arXiv preprint arXiv:1203.4697 (2012).

Anne-Marie Kermarrec, Erwan Le Merrer, Bruno Sericola, and Gilles Trédan. 2011. Second order centrality: Distributed assessment of nodes criticity in complex networks. Computer Communications 34, 5 (2011), 619-628.

Issa Khalil, Saurabh Bagchi, and Ness B Shroff. 2005. LITEWORP: A lightweight countermeasure for the wormhole attack in multihop wireless networks. In Proceedings of the International Conference on Dependable Systems and Networks (DSN'05).. IEEE, 612-621.

Philip Levis, Nelson Lee, Matt Welsh, and David Culler. 2003. TOSSIM: Accurate and scalable simulation of entire TinyOS applications. In Proceedings of the 1st International Conference on Embedded Networked Sensor Systems. ACM. 
P. Levis, S. Madden, J. Polastre, R. Szewczyk, K. Whitehouse, A. Woo, D. Gay, J. Hill, M. Welsh, E. Brewer, and D. Culler. 2005. TinyOS: An operating system for sensor networks. In Ambient Intelligence. Springer, Berlin.

Peter V. Marsden. 2002. Egocentric and sociocentric measures of network centrality. Soc. Netw. 24, 4 (2002), 407-422.

Sergio Marti, Thomas J Giuli, Kevin Lai, and Mary Baker. 2000. Mitigating routing misbehavior in mobile ad hoc networks. In Proceedings of the 6th Annual International Conference on Mobile Computing and Networking. ACM, 255-265.

Achille Messac, Amir Ismail-Yahaya, and Christopher A Mattson. 2003. The normalized normal constraint method for generating the Pareto frontier. Structural and Multidisciplinary Optimization 25, 2 (2003), 86-98.

Daniele Midi, Antonino Rullo, Anand Mudgerikar, and Elisa Bertino. 2017. Kalis: A system for knowledge-driven adaptable intrusion detection for the Internet of Things. In Proceedings of the IEEE 37th International Conference on Distributed Computing Systems (ICDCS'17).

A. Mishra, K. Nadkarni, and A. Patcha. 2004. Intrusion detection in wireless ad hoc networks. IEEE Wireless Communications 11, 1 (Feb 2004), 48-60. DOI : http://dx.doi.org/10.1109/MWC.2004.1269717

Asis Nasipuri and Kai Li. 2002. A directionality based location discovery scheme for wireless sensor networks. In Proceedings of the 1st ACM International Workshop on Wireless Sensor Networks and Applications. ACM.

Raymond T. Ng and Jiawei Han. 1994. Efficient and effective clustering methods for spatial data mining.

Nayot Poolsappasit, Rinku Dewri, and Indrajit Ray. 2012. Dynamic security risk management using bayesian attack graphs. IEEE Transactions on Dependable and Secure Computing 9, 1 (2012), 61-74.

Shahid Raza, Simon Duquennoy, Joel Höglund, Utz Roedig, and Thiemo Voigt. 2014. Secure communication for the Internet of Things a comparison of link-layer security and IPsec for 6LoWPAN. Security and Communication Networks 7, 12 (2014), 2654-2668.

Shahid Raza, Linus Wallgren, and Thiemo Voigt. 2013. SVELTE: Real-time intrusion detection in the Internet of Things. Ad Hoc Networks (2013).

Rodrigo Roman, Cristina Alcaraz, Javier Lopez, and Nicolas Sklavos. 2011. Key management systems for sensor networks in the context of the Internet of Things. Comput. Electr. Eng. 37, 2 (2011), 147-159.

Antonino Rullo, Daniele Midi, Edoardo Serra, and Elisa Bertino. 2017a. A game of things: Strategic allocation of security resources for IoT. In Procedings of the ACM/IEEE 2nd International Conference on Internet-of-Things Design and Implementaion (IoTDI'17). ACM/IEEE.

Antonino Rullo, Edoardo Serra, Elisa Bertino, and Jorge Lobo. 2017b. Shortfall-based optimal placement of security resources for mobile IoT scenarios. In European Symposium on Research in Computer Security (ESORICS'17). Springer, Berlin.

Edoardo Serra, Sushil Jajodia, Andrea Pugliese, Antonino Rullo, and V. S. Subrahmanian. 2015. Pareto-optimal adversarial defense of enterprise systems. ACM Trans. Inf. Syst. Secur. 17, 3 (2015), 11.

Kalpana Sharma and M. K. Ghose. 2010. Wireless sensor networks: An overview on its security threats. IFCA Special Issue on Mobile Ad-Hoc Networks MANETs. 42-45.

Chanatip Tumrongwittayapak and Ruttikorn Varakulsiripunth. 2009. Detecting sinkhole attack and selective forwarding attack in wireless sensor networks. In Proceedings of the International Conference on Intelligent Circuits and Systems (ICICS'09).

András Varga and others. 2001. The OMNeT++ discrete event simulation system. In Proceedings of the European Simulation Multiconference (ESM’01).

Heinrich von Stackelberg, Damien Bazin, Rowland Hill, and Lynn Urch. 2010. Market Structure and Equilibrium. Springer, Berlin.

Dazhi Zhang and Donggang Liu. 2010. DataGuard: Dynamic data attestation in wireless sensor networks. In Proceedings of the IEEE International Conference on Dependable Systems and Networks (DSN'10). IEEE.

Liang Zhou and Han-Chieh Chao. 2011. Multimedia traffic security architecture for the Internet of Things. IEEE Netw. 25, 3 (2011), 35-40.

Quanyan Zhu, Linda Bushnell, and Tamer Basar. 2012. Game-theoretic analysis of node capture and cloning attack with multiple attackers in wireless sensor networks. In Proceedings of the IEEE Conference on Decision and Control (CDC'12). IEEE, 3404-3411.

Quanyan Zhu, Husheng Li, Zhu Han, and Tamer Basar. 2010. A stochastic game model for jamming in multi-channel cognitive radio systems. In Proceedings of the IEEE International Conference on Communications (ICC'10).

Alliance Zigbee. 2006. Zigbee specification. ZigBee document 053474r13 (2006).

Received February 2017; revised June 2017; accepted August 2017 